\title{
Mechanisms of Targeting the MDM2- p53-FOXM1 Axis in Well-Differentiated Intestinal Neuroendocrine Tumors
}

\author{
Franziska Briest $^{\mathrm{a}-\mathrm{c}}$ Irina Grass ${ }^{\mathrm{a}, \mathrm{b}}$ Dagmar Sedding ${ }^{\mathrm{a}, \mathrm{d}}$ Markus Möbs $^{\mathrm{e}}$ \\ Friederike Christen $^{\text {a,d }}$ Joana Benecke ${ }^{f}$ Karolin Fuchs $^{g}$ Stefanie Mende ${ }^{e}$ \\ Daniel Kaemmerer $^{\text {h }}$ Jörg Sänger ${ }^{i} \quad$ Almut Kunze $^{i} \quad$ Christina Geisler $^{j}$ \\ Helma Freitag ${ }^{a, k} \quad$ Florentine Lewens $^{a} \quad$ Lina Worpenberg $^{a, b}$ \\ Sara Iwaszkiewicz ${ }^{a, b} \quad$ Britta Siegmund $^{a} \quad$ Wolfgang Walther $^{g, 1}$ \\ Michael Hummel ${ }^{\mathrm{e}}$ Patricia Grabowski ${ }^{\mathrm{a}, \mathrm{c}, \mathrm{k}}$ \\ a Department of Gastroenterology, Infectious Diseases, Rheumatology CC13, Charité - Universitätsmedizin \\ Berlin, and ${ }^{b}$ Department of Chemistry and Biochemistry, Freie Universität (FU) Berlin, Berlin, ' Department of \\ Gastroenterology and Endocrinology, Zentralklinik Bad Berka GmbH, Bad Berka, ${ }^{d}$ Institute of Biology, \\ Humboldt-Universität Berlin, ${ }^{\mathrm{e}}$ Institute of Pathology, Charité - Universitätsmedizin Berlin, ${ }^{\mathrm{f}}$ Institute of \\ Biotechnology, Technische Universität Berlin, and ${ }^{\mathrm{g}} \mathrm{EPO} \mathrm{GmbH}$ Berlin-Buch, Berlin, ${ }^{\mathrm{h}}$ Department of General and \\ Visceral Surgery, Zentralklinik Bad Berka GmbH, and 'Institute of Pathology, Bad Berka, ${ }^{\text {, Department of }}$

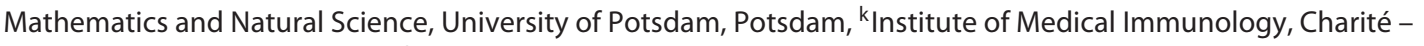 \\ Universitätsmedizin Berlin, and 'Experimental and Clinical Research Center (ECRC), Charité University Medicine \\ Berlin and Max Delbrück Center for Molecular Medicine, Berlin, Germany
}

\section{Keywords}

Neuroendocrine tumors · Signaling · MDM2 - p53 · FOXM1 .

Targeted therapy

\begin{abstract}
Background/Aims: The tumor suppressor p53 is rarely mutated in gastroenteropancreatic neuroendocrine neoplasms (GEP-NEN) but they frequently show a strong expression of negative regulators of $\mathrm{p} 53$, rendering these tumors excellent targets for a p53 recovery therapy. Therefore, we analyzed the mechanisms of a p53 recovery therapy on intestinal neuroendocrine tumors in vitro and in vivo. Methods: By West-
\end{abstract}

ern blot and immunohistochemistry, we found that in GEPNEN biopsy material overexpression of MDM2 was present in intestinal NEN. Therefore, we analyzed the effect of a small-molecule inhibitor, nutlin-3a, in p53 wild-type and mutant GEP-NEN cell lines by proliferation assay, flow cytometry, immunofluorescence, Western blot, and by multiplex gene expression analysis. Finally, we analyzed the antitumor effect of nutlin-3a in a xenograft mouse model in vivo. During the study, the tumor volume was determined. $R \boldsymbol{e}$ sults: The midgut wild-type cell line KRJ-I responded to the treatment with cell cycle arrest and apoptosis. By gene expression analysis, we could demonstrate that nutlins reactivated an antiproliferative $\mathrm{p} 53$ response. KRJ-I-derived xeno-

\section{KARGER}

(c) 2017 S. Karger AG, Basel

E-Mail karger@karger.com

www.karger.com/nen
Franziska Briest

Department of Gastroenterology, Infectious Diseases, Rheumatology CC13

Charité - Universitätsmedizin Berlin

DE-12200 Berlin (Germany)

E-Mail franziska.briest@ charite.de 
graft tumors showed a significantly decreased tumor growth upon treatment with nutlin-3a in vivo. Furthermore, our data suggest that MDM2 also influences the expression of the oncogene FOXM1 in a p53-independent manner. Subsequently, a combined treatment of nutlin-3a and cisplatin (as chemoresistance model) resulted in synergistically enhanced antiproliferative effects. Conclusion: In summary, MDM2 overexpression is a frequent event in p53 wild-type intestinal neuroendocrine neoplasms and therefore recovery of a p53 response might be a novel personalized treatment approach in these tumors.

(c) 2017 S. Karger AG, Basel

\section{Introduction}

Gastroenteropancreatic neuroendocrine neoplasms (GEP-NEN) are a rare family of heterogeneous tumors, which are predominantly characterized by the secretion of hormones and signaling peptides and generally by a slow growing behavior. They are localized in the gastrointestinal system or the pancreas and are often diagnosed only when they have already entered the metastatic phase, due to the unobtrusive growth of the primary site [1].

Low-grade GEP-NEN are not susceptible to chemotherapy, as they have a low proliferation rate. Targeted therapy approaches show subtype-specific success, and, especially for midgut NEN, more effective medical treatment options are necessary [2].

The molecular signaling of GEP-NEN is characterized by a strong activity of various kinases, especially of growth factor-dependent pathways, such as the PI3K-mTOR pathway or the MAPK signaling [3]. Few driver mutations are known to date, but most of them are not actionable.

This study is focused on the therapeutic potential of targeting the MDM2-p53 interaction in the intestinal tumor subgroup. The E3 ubiquitin ligase MDM2 is a crucial negative regulator of the tumor suppressor protein $\mathrm{p} 53$. The latter is involved in the regulation of a multitude of response mechanisms to cellular stress, and its gene, TP53, is frequently mutated in several cancers [4].

Previous studies have demonstrated that GEP-NEN are rarely mutated in TP53 [5-8]. Nevertheless, in pancreatic neuroendocrine tumors (NEN), the p53 signaling is affected due to an intense positive regulator gene silencing and overexpression of negative regulators, in particular MDM2, MDM4, and WIP1 [9]. Furthermore, activation of AKT, promoter methylation of RASSF1A, and $D A X X$ mutation might influence the stability of MDM2
[10-13]. Data referring to MDM2 in the intestinal tumor subgroup is still lacking. If MDM2 is also overexpressed in the intestinal subgroup, recovery of $\mathrm{p} 53$ function could potentially be an effective therapeutic approach in this entity [14]. In addition, tumor suppressor activity of p53 has been described to be involved in the regulation of FOXM1 $[15,16]$, a critical downstream target and oncogene in many solid tumors, and actionable target in gastrointestinal NEN [17].

The primary aim of this study is to therapeutically recover a p53 wild-type response in GEP-NEN cells, which is characterized by the regulation of p53 targets (such as p21 or GADD45), and induction of cell cycle arrest and apoptosis [4]. The secondary aim is to evaluate the treatment response on FOXM1 expression, which is involved in gastrointestinal NEN progression [17] and crucially regulates DNA repair mechanisms [18]. The latter aspect might be relevant for combined chemotherapeutic-p53 recovery approaches.

Therefore, we assess MDM2-p53 interaction inhibition in monotherapy and combination for GEP-NEN treatment in vitro and in vivo and describe important signaling changes in the p53 network, including FOXM1 regulation.

\section{Material and Methods}

\section{Patients and Samples}

In this prospective study, 34 fresh frozen tissue samples of GEPNEN, derived from 23 patients, were collected at the Department of General and Visceral Surgery at the Zentralklinik Bad Berka $\mathrm{GmbH}$ within the years 2009-2013. The largest subgroup of the tumor specimens were intestinal tumors $(n=25)$. Biobanking was accomplished with an institutional review board approval for guidelines and ethical procedures. The NEN diagnosis was based on the immunohistochemical characterization of chromogranin A and synaptophysin expression, on proliferation index determination (Ki-67) and on morphological criteria according to the WHO 2010 grading classification. All G3 tumors have been analyzed for TP53 mutations by next-generation sequencing. One G3 tumor was bearing a c. $844>\mathrm{T}$ R $282 \mathrm{~W}$ mutation in exon 8 of TP53, but the confirmed allele frequency was as low as $6 \%$ of all tumor cells. Frozen tissue and FFPE (formalin-fixed and paraffin-embedded) specimens were hematoxylin- and eosin-stained and analyzed for tumor cell ratio and morphological features. Frozen samples consisting of more than $80 \%$ tumor cells were included. All frozen samples were mechanically homogenized and lysed in NP-40 buffer.

FFPE specimens were immunohistochemically stained as described below. Histopathological and clinical data of the patients and tumor samples can be found in Table 1; the distribution of analyzed primary tumors and metastases per patient can be found in online suppl. Table 1 (frozen tissue) and suppl. Table 2 (FFPE specimens) (see www.karger.com/doi/10.1159/000481506 for all online suppl. material).
Briest et al. 
Table 1. Clinicopathological data of analyzed GEP-NEN primary tumor material

\begin{tabular}{|c|c|c|}
\hline Tissue type & Primary tumors & Metastases \\
\hline \multicolumn{3}{|l|}{ GEP-NEN } \\
\hline Total $(n=34)$ & 14 & 20 \\
\hline \multicolumn{3}{|l|}{ Grading } \\
\hline G1 & 9 & 14 \\
\hline G2 & 2 & 4 \\
\hline G3 & 3 & 2 \\
\hline \multicolumn{3}{|l|}{ Localization of the primary } \\
\hline Pancreatic & 4 & 4 \\
\hline Intestinal (see below) & 10 & 15 \\
\hline CUP & - & 1 \\
\hline \multicolumn{3}{|l|}{ Localization of metastases } \\
\hline Lymph node & - & 5 \\
\hline Distant & - & 15 \\
\hline \multicolumn{3}{|l|}{ Gender } \\
\hline Male & 8 & 8 \\
\hline Female & 6 & 12 \\
\hline \multicolumn{3}{|l|}{ Age at diagnosis, years } \\
\hline Min. & 42 & 53 \\
\hline Max. & 77 & 79 \\
\hline Average & 61.7 & 64.5 \\
\hline \multicolumn{3}{|l|}{ Intestinal NEN } \\
\hline Total $(n=25)$ & 10 & 15 \\
\hline \multicolumn{3}{|l|}{ Grading } \\
\hline G1 & 7 & 13 \\
\hline G2 & 1 & 0 \\
\hline G3 & 2 & 2 \\
\hline \multicolumn{3}{|c|}{ Localization of the primary } \\
\hline Ileum & 6 & 14 \\
\hline Duodenum & 2 & 0 \\
\hline Colon & 2 & 1 \\
\hline \multicolumn{3}{|l|}{ Localization of metastases } \\
\hline Lymph node & - & 4 \\
\hline Distant & - & 11 \\
\hline \multicolumn{3}{|l|}{ Gender } \\
\hline Male & 6 & 6 \\
\hline Female & 4 & 9 \\
\hline \multicolumn{3}{|l|}{ Age at diagnosis, years } \\
\hline Min. & 42 & 55 \\
\hline Max. & 77 & 79 \\
\hline Average & 63.0 & 65.6 \\
\hline
\end{tabular}

Data are provided for all used GEP-NEN fresh frozen tumor samples $(n=34)$ and further specified for the included 25 intestinal NEN tumors.

\section{Cell Lines}

The following GEP-NEN cell lines were used for in vitro experiments: pancreatic BON [19] and QGP-1 [20] (obtained from the Japanese Collection of Research Bioresources), ileal KRJ-I [21], and colonic LCC-18 [22]. BON cells were a generous gift from C.M. Townsend (University of Texas, Galveston). KRJ-I midgut neuroendocrine tumor (NET) cells were generated by R. Pfragner
(Medical University of Graz) and kindly provided by I. Modlin (Yale University, New Haven).

Cells are cultured and handled as previously published [17]. All cell lines were authenticated (if indicated as unique) by genetic short tandem repeat typing at the DSMZ, Braunschweig, Germany, in 2012, 2013, and 2015. Cells were not passaged for more than 20 passages after receipt. Cells were further tested periodically for maintained cell line-specific expression of neuroendocrine and differentiation markers (chromogranin A, synaptophysin, cytokeratin, vimentin, syntaxin) by immunofluorescence microscopy.

\section{Deep Sequencing of TP53}

Cell lines were cultured, harvested, and genomic DNA was isolated using the DNeasy Blood and Tissue Kit (Qiagen, Venlo, the Netherlands) according to the manufacturer's instructions. DNA was isolated from FFPE material using the Maxwell ${ }^{\circledR}$ RSC DNA FFPE Kit with minor protocol modifications.

Multiplex-PCR based amplicon high-throughput sequencing was performed using the Ion Torrent PGM system (Life Technologies; Carlsbad, CA, USA) according to the manufacturer's recommendations. Briefly, $10 \mathrm{ng}$ of genomic DNA was used for library construction with the Ion AmpliSeq Colon and Lung Cancer Panel v2 and the Ion AmpliSeq Library Kit 2.0 (Life Technologies). The library quantification was carried out on the 7900HT Fast RealTime PCR System (Applied Biosystems) employing the Ion Library TaqMan Quantitation Kit (Life Technologies). Emulsion PCR and subsequent enrichment were realized with the help of the OneTouch 2 system (Life Technologies). For high-throughput sequencing, the $318 \mathrm{v} 2$ chip was utilized as recommended by the manufacturer. Data analysis was performed using the Torrent Suite Software v4.2 (Life Technologies). Amplicons covering the genomic region of TP53 were as follows: chr17:7579854-7579960, chr17: 7579351-7579485, chr17:7578517-7578601, chr17: 7578353-7578483, chr17:7578181-7578298, chr17:7577509_ 7577612, chr17:7577016-7577151, chr17:7573924-7574035.

\section{Sanger Sequencing of TP53 Exon 4}

The chromosomal region chr17:7.579.343 to chr17:7.579.503 (hg 19) was amplified from 50 ng of genomic DNA with Amplitaq gold DNA polymerase in gold buffer supplemented with $1.5 \mathrm{mM}$ $\mathrm{MgCl}_{2}$ (Applied Biosystems) at an annealing temperature of $56^{\circ} \mathrm{C}$. After purification, direct sequencing was performed using the BigDye Terminator v1.1 Cycle Sequencing Kit (Applied Biosystems) and subsequent capillary electrophoresis on an ABI 3130 Genetic Analyzer. Primers were purchased from TIB Molbiol. Primer sequences were as follows: TP53_ex4_s: $5^{\prime}$-GAAGACCCAGGTCCAGAT-3', TP53_ex4_as: 5'-GACTTGGCTGTCCCAGAA-3 ${ }^{\prime}$ (generated from Primer3 v1.1.4, purchased from Metabion International AG, Planegg, Germany).

\section{Fluorescence in situ Hybridization}

Cells were grown, harvested, and fixed after cytospin in buffered formalin overnight. Cytospins were dried for 2 min in 70, 80, and $90 \%$ ethanol at $56^{\circ} \mathrm{C}$. Histology FISH Accessory Kit 7599 (Dako Deutschland GmbH, Hamburg, Germany) was applied for pretreating and washing according to the manufacturer's instructions. For MDM2 fluorescence in situ hybridization (FISH) we used the following probes: Vysis LSI MDM2 SpectrumOrange probe (MDM2) and CEP 12 SpectrumGreen probe (chromosome enumeration probe: 12p11.1-q11 Alpha Satellite DNA) in LSI/ 
WCP $^{\circledR}$ hybridization buffer (all Abbott Molecular, Abbott Park, Illinois, USA). Probes were denaturized at $80^{\circ} \mathrm{C}$ for $10 \mathrm{~min}$, hybridized at $37^{\circ} \mathrm{C}$ overnight and DAPI counterstained. FISH signals were analyzed with Zeiss Axio-Imager Z1 and ISIS software v5.3.1 (MetaSystems GmbH, Altlussheim, Germany).

\section{Immunofluorescence}

Cells were grown on cover slips or spotted by cytospin and fixed with 1:1 methanol/acetone. Cells were blocked in $2 \%$ milk buffer for $30 \mathrm{~min}$ and washed. Primary antibodies for MDM2, p53, and FOXM1 (Santa Cruz Biotechnology Inc., Dallas, TX, USA) were incubated 1:30-1:50 in a wet chamber with PBS containing $0.1 \% \mathrm{BSA}$ and $0.5 \%$ Triton X overnight at $4^{\circ} \mathrm{C}$. After washing, secondary antibodies Alexa fluor 488 goat anti-mouse and Alexa fluor 594 goat anti-rabbit (Life Technologies) were applied 1:200 in PBS containing $0.1 \%$ BSA and $0.5 \%$ Triton X for $1 \mathrm{~h}$. After washing, cells were fixed for 2 min in $96 \%$ ethanol and embedded with Roti $^{\circledR}$-Mount FluorCare DAPI (Carl Roth GmbH, Karlsruhe, Germany).

\section{Immunohistochemistry}

Paraffin-embedded tumor specimens of 18 out of 23 patients were further analyzed by immunohistochemistry (online suppl. Table 2). Immunohistochemistry was performed by the avidinbiotin-peroxidase complex method. Briefly, 4- $\mu$ m paraffin sections were transferred to glass slides and dried overnight. After deparaffination and rehydration, the endogenous peroxidase activity was blocked with $0.5 \% \mathrm{H}_{2} \mathrm{O}_{2}$ (in distilled water) for $30 \mathrm{~min}$. Nonspecific binding was reduced by incubation with normal horse serum for $20 \mathrm{~min}$. The following antibodies were used: p53, 1:50, Mouse Klon 14/95, Dianova, Hamburg; FoxM1 (C-20), 1:100, rabbit, polyclonal, Santa Cruz Biotechnology; MDM2 (SMP-14), 1:100, mouse, MW, 0.01 M citrate, pH 6.0, Santa Cruz Biotechnology. The specific staining was enhanced by heat antigen retrieval. Sections were immersed in $0.01 \mathrm{~mol} / \mathrm{L}$ citrate buffer, $\mathrm{pH}$ 6.0, and irradiated in a microwave oven at $520 \mathrm{~W}$ for $4 \mathrm{~min}$ and $400 \mathrm{~W}$ for $12 \mathrm{~min}$. The sections were kept at room temperature for $15 \mathrm{~min}$. After washing with PBS, the incubation with the primary antibody was carried out in a humidified chamber at $4^{\circ} \mathrm{C}$ overnight. Thereafter, the sections were rinsed carefully with PBS and incubated with a biotinylated horse anti-mouse antibody in case of p53 and MDM2 detection or a biotinylated horse anti-rabbit antibody in case of the detection of FoxM1 in a dilution of 1:200 in PBS, supplemented with $0.1 \%$ BSA for 30 min. After 3 washes with PBS, sections were incubated for $30 \mathrm{~min}$ with a preformed macromolecular complex consisting of avidin and biotinylated peroxidase which still retains biotin-binding sites. All steps except for the incubation with the primary antibody were carried out at room temperature. The staining reaction was performed by incubation with a solution

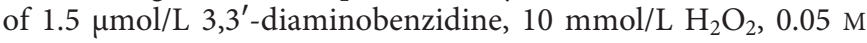
Tris-buffered saline, $\mathrm{pH} 7.6$, at $25^{\circ} \mathrm{C}$ for $5 \mathrm{~min}$. After counterstaining in 2\% Harry's hematoxylin for $5 \mathrm{~min}$, the sections were dehydrated with ethanol. Slides were incubated with xylene and then mounted with Canada balsam.

The percentage of cells with FOXM1 nuclear immunoreactivity was determined as: $0=$ no signal; $1=\leq 10 \% ; 2=11-50 \% ; 3=$ 51-80\%; $4=>80 \%$. The score was dichotomized as follows: no FOXM1 expression, 0 or 1 ; FOXM1 expression, $\geq 2$. The percentage of cells with MDM2 nuclear expression was determined analogously to FOXM1 and multiplied by the intensity of the staining
( 1 = weak; 2 = intermediate; 3 = strong). The resulting score was dichotomized with a cutoff of $6(<6$, weak expression; $\geq 6$, strong expression).

\section{Real-Time PCR}

RNA was isolated using the Qiagen RNeasy RNA isolation kit, quantified with Nanodrop 2000, and DNA digesting was carried out using $0.1 \mu \mathrm{L} / 100$ ng RNA DNase I peqGOLD (Peqlab, VWR International $\mathrm{GmbH}$, Erlangen, Germany). 1,000 ng RNA was translated into cDNA using the High-Capacity RNA-to-cDNA ${ }^{\mathrm{TM}}$ kit according to the manufacturer's instructions (Thermo Fisher, Carlsbad, CA, USA). Real-time PCR was carried out with 25 ng cDNA with the StepOne real-time PCR system (Thermo Fisher) and analyzed with StepOne v2.3 software and MS Excel using the $\Delta \Delta \mathrm{C}_{\mathrm{t}}$ method. A figure was prepared with Prism 6 software. The PCR primers were used as follows: FOXM1 forward 5'-GGA GCA GCG ACA GGT TAA GG-3', FOXM1 reverse $5^{\prime}$-GTT GAT GGC GAA TTG TAT CAT GG-3', FOXM1A forward 5'-TGG GGA ACA GGT GGT GTT TGG-3', FOXM1A reverse $5^{\prime}$-GCT AGC AGC ACT GAT AAA CAA AG-3', FOXM1B forward 5'-CCA GGT GTT TAA GCA GCA GA-3', FOXM1B/C reverse $5^{\prime}$-TCC TCA GCT AGC AGC ACC-3', FOXM1C forward 5'-CAA TTG CCC GAG CAC TTG GAA TCA-3', p21 forward 5'-CCT GGC TGA CTT CTG CTG TCT-3', p21 reverse $5^{\prime}$-CGG CGT TTG GAG TGG TAG A-3', MDM2 forward $5^{\prime}$-GGC TCT GTG TGT AAT AAG GGA GA-3', MDM2 reverse $5^{\prime}$-GGA CTG CCA GGA CTA GAC TTT G-3', GUS (housekeeper) forward 5' -GAA AAT ATG TGG TTG GAG AGC TCA TT-3', GUS reverse 5' -CCG AGT GAA GAT CCC CTT TTT A-3' (all: Metabion).

\section{Western Blot}

SDS-PAGE and Western blot of NP-40-lysed primary tumor or cell line material (cells were synchronized in $0.01 \%$ FBS for $24 \mathrm{~h}$ before treatment) was performed using a standard protocol and documented by Ponceau S staining. Primary antibodies were obtained from Cell Signaling Technology Inc., Danvers, MA, USA (pan-actin: D18C11, phospho-histone H3 (Ser10), BID, phosphoFoxM1 (Ser35), phospho-p70 S6 kinase (Thr389), p70S6 kinase, cleaved PARP, phospho-AKT (Ser473), AKT, phospho-FOXO1 (Thr24)/FOXO3a (Thr32), SKP2, caspase 8, caspase 9, phosphohistone H2AX (Ser139), phospho-p53 (Ser20), and p53), Santa Cruz Biotechnology Inc., Dallas, TX, USA (FOXM1: C-20 and G-5, RAD51, p53, FEN1, MDM2, BAX, and p21), GeneTex Inc., Irvine, CA, USA (GAPDH), Sigma Aldrich, St. Louis, MO, USA ( $\beta$-tubulin: TUB2.1), Abcam Cambridge, UK (aurora A), BD Biosciences (FADD, TRADD), and secondary antibodies were obtained from Dako Deutschland GmbH, Hamburg, Germany (swine anti-rabbit IgG-HRP and goat anti-mouse IgG-HRP). Antibody binding was documented by Fujifilm LAS-4000 luminescent image analyzer using ECL ${ }^{\mathrm{TM}}$ prime Western blotting detection reagent (Amersham $^{\mathrm{TM}} \mathrm{GE}$ healthcare). For reprobing, membranes were treated with acidic glycine buffer as published [17]. Chemiluminescence signals were densitometrically analyzed with Multi Gauge V3.1. Values of $\geq 3$ independent experiments were normalized to internal controls, and mean values were statistically assessed by using IBM SPSS Statistics v22.

\section{Statistical Analyses}

For analyses of the Western blot densitometry data (primary and cell line material), Mann-Whitney U or Kruskal-Wallis tests 
were applied; statistical analysis was performed using IBM SPSS Statistics v22 software. Multivariate analysis was performed by forward and reverse stepwise linear regression using SPSS 22. The flow cytometry and WST-1 analyses of nutlin treatment were analyzed using Prism 6 with 1-way ANOVA, 2-way ANOVA, Kruskal-Wallis or Whitney U test, respectively, and Dunnett, Sidak, or Bonferroni correction for multiple testing. The choice was dependent on the structure of the data; low numbers of samples $(<5)$ were always analyzed with nonparametric tests, parametric tests were chosen after testing for normality in data with $n \geq 5$. Normality was assumed after a Kolmogorov-Smirnow test. Linear trend was analyzed using ANOVA with a post test for linear trend. The differential expression results of the gene expression analysis were analyzed with nSolver ${ }^{\circledR}$ v2.5 software (Nanostring ${ }^{\circledR}$ technologies) using first principal component analysis and regression analysis with and without the Benjamini-Yekutieli procedure. The gene ontology (GO) overrepresentation analysis was performed with the http://pantherdb.org/ database using the PANTHER overrepresentation test (release 20170413) based on the "PANTHER GOslim biological process" annotation data set (PANTHER version 11.1 released October 24, 2016) with Bonferroni correction for multiple testing.

All differences were considered to be significant with $\alpha=0.05$. Immunohistochemistry was analyzed by SPSS 22 applying Fisher's exact test.

\section{GEP-NEN Cell Line Treatment with Nutlins}

Nutlin-3 (CAS No. 890090-75-2) was obtained from Santa Cruz Biotechnologies; nutlin-3a (CAS No. 675576-98-4) was obtained from Selleck Chemicals, everolimus from Cell Signaling, and siomycin A from Sigma Aldrich. Substances were dissolved in DMSO. WST-1 proliferation assays were performed according to the manufacturer's instructions in quintuplets after treatment with decreasing concentrations of the substances. The WST-1 proliferation reagent was obtained from Roche, Basel, Switzerland. Cell density was colorimetrically quantified with a multiwell spectrophotometer (TECAN sunrise ${ }^{\mathrm{TM}}$ ) and analyzed with MS Excel 2013 and Prism 6.

\section{Cell Cycle Analysis}

For mitotic index flow cytometry, cells were treated with $5 \mu \mathrm{M}$ nutlin-3a and stained following a previously published protocol [17]. For JC-1 staining, cells were harvested after treatment with $5 \mu \mathrm{M}$ nutlin-3a, $10 \mu \mathrm{M}$ cisplatin + DMSO or $5 \mu \mathrm{M}$ nutlin- $3 \mathrm{a}+10$ $\mu \mathrm{M}$ cisplatin and suspended in PBS. Positive controls were incubated 1:500 with the generic mitochondrial membrane depolarizer carbonylcyanide $m$-chlorophenylhydrazone. All samples (negative controls excluded) were incubated with 1:500 JC-1 for $30 \mathrm{~min}$ at $37^{\circ} \mathrm{C}$. Flow cytometry was conducted with FACSCalibur (Becton Dickinson) by BD Cell Quest Pro software and analyzed with FlowJo 8.7 software, Prism 6, and IBM SPSS Statistics 22.

\section{nCounter ${ }^{\circledR}$ Multiplex Gene Expression Analysis}

Cells were treated in duplets with $5 \mu \mathrm{M}$ nutlin-3a versus DMSO for $60 \mathrm{~h}$. RNA was isolated with an RNeasy Mini Kit (Qiagen) according to the manufacturer's instructions. RNA was measured using Nanodrop (Thermo Fisher Scientific). 60 ng RNA was applied in the PanCancer ${ }^{\circledR}$ pathway (Nanostring ${ }^{\circledR}$ technologies, Seattle, WA, USA) analysis according to the manufacturer's instruction. The assay was analyzed with nSolver ${ }^{\circledR}$ v2.5 (based on R v3.1.1). Raw data were analyzed with the 1 st PC method (nSolver ${ }^{\circledR}$ v2.5) and normalized to the following housekeeping genes: HDAC3, SAP130, DDX50, PIAS1, FCF1, PRPF38A, TLK2, USP39, EIF2B4, ACAD9, CNOT10, EDC3, NOL7, C10orf76, DNAJC14, CNOT4, TTC31, ZC3H14, PIK3R4, AGK, ZNF384, FTSJ2, ZNF143, MRPS5, MTMR14, SLC4A1AP, SF3A3, AMMECR1L, and ERCC3 for KRJ-I.

\section{Combination Therapy of GEP-NEN Cell Lines}

Cells were seeded in quintuplets in 96-well plates. Cells were treated with $20 \mu \mathrm{M}$ nutlin-3a and $100 \mu \mathrm{M}$ cisplatin in 1 - to 128 -fold dilution or respective monotherapies, to obtain dose-response curves as recommended by the Chou-Talalay method [23]. Cisplatin was obtained from the Charité Berlin dispensary. The WST-1 proliferation assay (Roche, Basel, Switzerland) was performed and colorimetrically quantified. Data were analyzed by Prism 6, CompuSyn $1.0[24]$.

\section{In vitro RNA Interference Knockdown}

Cells were transfected with $30-50 \mathrm{pmol} / \mathrm{mL}$ small interfering RNA (siRNA) diluted in lipofectamine 3000 (Life Technologies, Carlsbad, CA, USA) according to the manufacturer's instructions for $24 \mathrm{~h}$.

siRNAs used were: Qiagen FlexiTube siRNA against human MDM2 (Hs_MDM2_10 and Hs_MDM2_9) and against FOXM1 (Hs_FOXM1_6). Alternative FOXM1 siRNA was: esiRNA against FOXM1 by Sigma (EHU124431: NCBI reference sequences: NM_021953, NM_202002, NM_202003). Negative controls were: Qiagen AllStars Negative Control siRNA and Sigma Mission ${ }^{\circledR}$ siRNA Universal negative control \#1. Positive control: AllStars Hs Cell Death Control siRNA (Qiagen).

\section{Animal Model and Treatment}

All animal experiments were carried out in accordance with the German Animal Welfare Act as well as the United Kingdom Coordinating Committee on Cancer Research; approval ID: G 0030/15 (Landesamt für Gesundheit und Soziales Berlin, Germany).

Fresh KRJ-I tumor fragments were transplanted subcutaneously into the left flank of 8 anesthetized female NOG mice per treatment group. The mice were observed for health condition during the study and maintained under sterile and controlled conditions $\left(22^{\circ} \mathrm{C}, 50 \%\right.$ relative humidity, 12 -h light-dark cycle, autoclaved food and bedding, acidified drinking water). The tumor growth was monitored, and treatment was started at palpable tumor size $\left(0.09 \mathrm{~cm}^{3}\right) .20 \mathrm{mg} / \mathrm{kg}$ nutlin-3a (or vehicle control) was applied intravenously every second day. Tumor growth was measured in 2 dimensions with a caliper. Tumor volumes (TV) were calculated by the formula: $\mathrm{TV}=\left(\right.$ width $^{2} \times$ length $) \times 0.5$.

\section{Results}

\section{MDM2 and FOXM1 Expression Is Connected with Metastasis in GEP-NEN}

The high incidence of MDM2 overexpression in metastatic pancreatic NEN is well studied [9]. Due to limited data concerning MDM2 expression in intestinal tumors, 


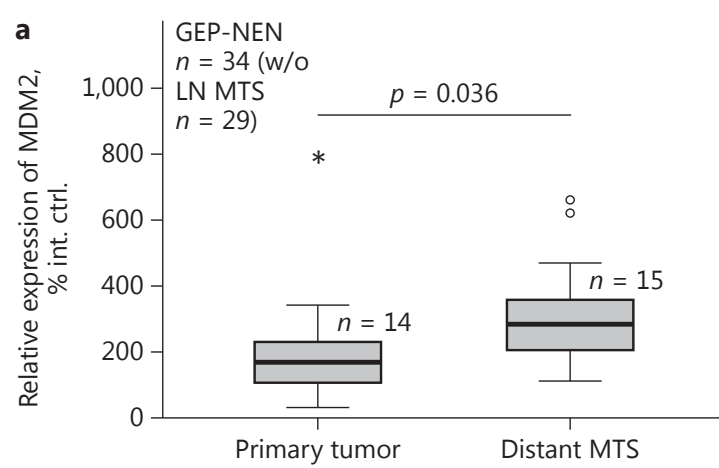

C

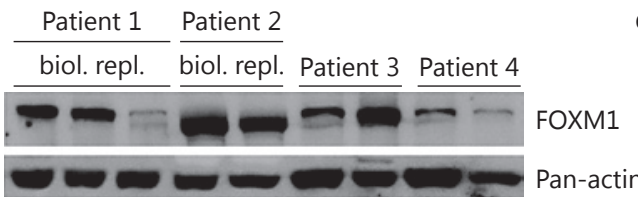

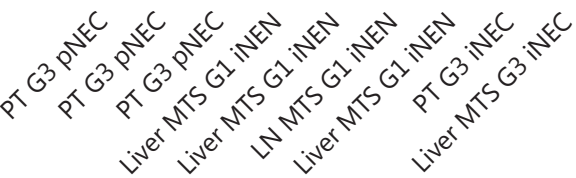

e

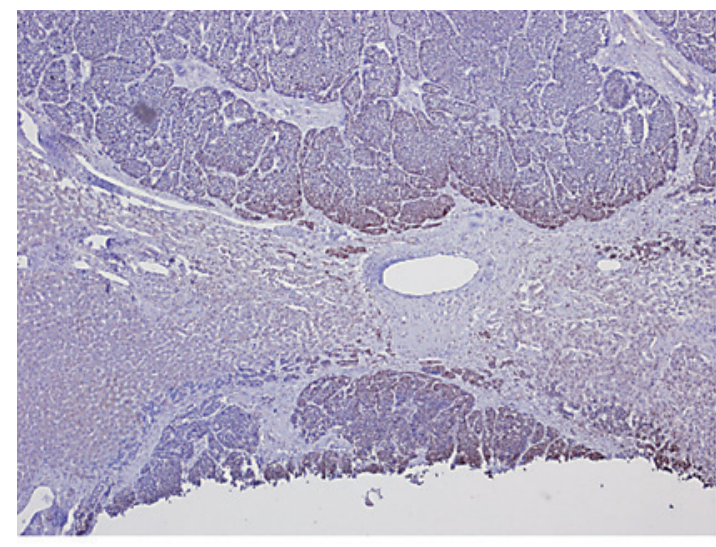

MDM2 40x

g

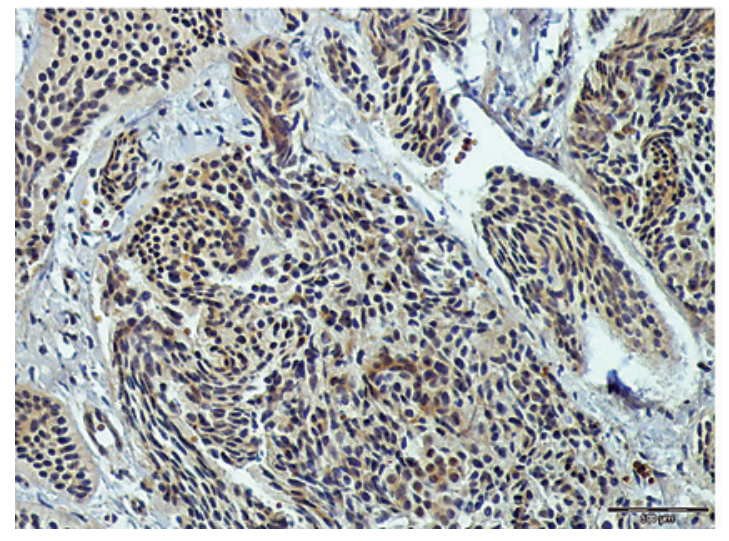

FOXM1

d
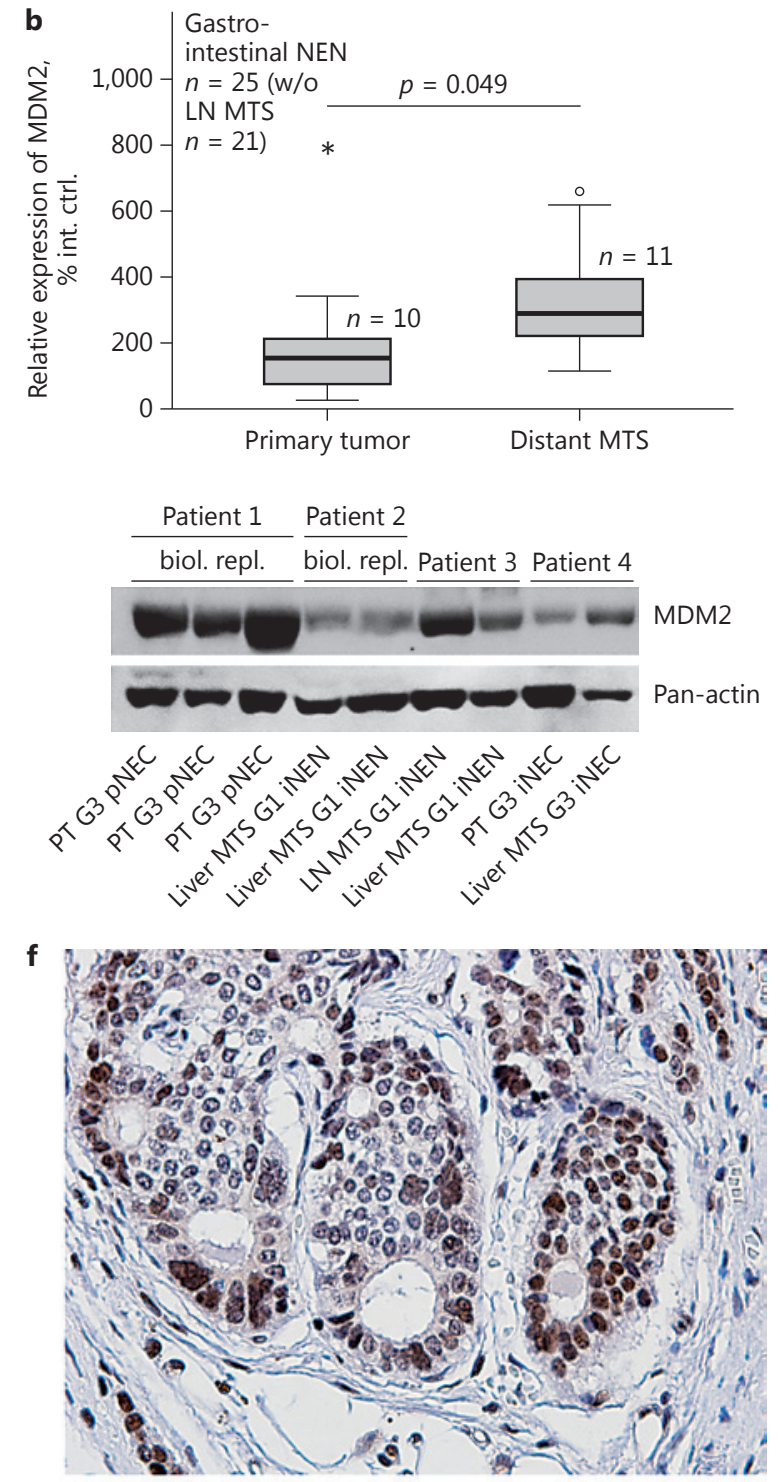

MDM2 400x

h

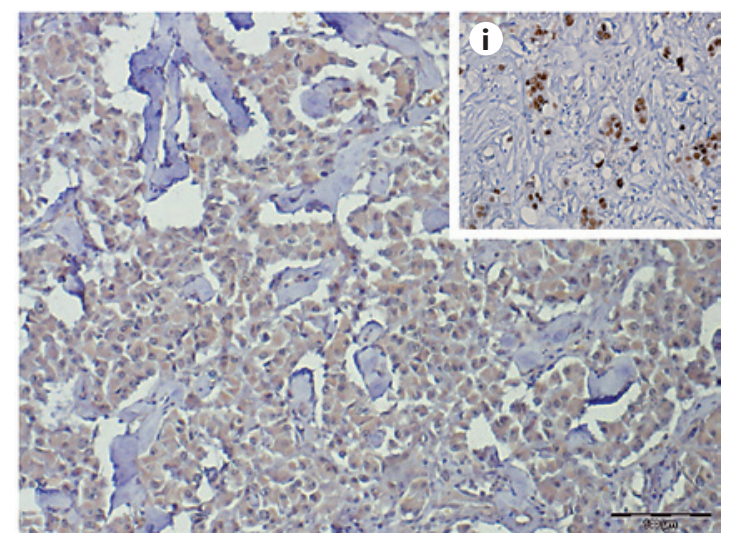

p53 
we had to verify the incidence of high MDM2 expression also in intestinal NEN. We therefore prospectively analyzed 34 frozen tumor and metastasis GEP-NEN biopsies of 23 patients (Table 1; online suppl. Table 1).

We found that MDM2 expression was significantly related to metastasis, as its relative expression was significantly higher in distant metastatic tumor samples than in primary tumors (Fig. 1). This applies to all 34 GEP-NEN in general (Fig. 1a; $p=0.036$ ) and to the intestinal subgroup ( 25 of 34 ; Fig. $1 \mathrm{~b} ; p=0.049$ ). Lymph node metastases exhibited an intermediate expression of MDM2 (higher than in primary tumors, lower than in distant metastases; data not significant and not shown). These results indicate a similar predominance of MDM2 in progressive intestinal NEN as published for pancreatic NEN, unless the results should be confirmed in a larger number of cases.

We further densitometrically analyzed FOXM1 expression of primary tumors versus metastasis in this tumor collection $(n=18)$. We found FOXM1 elevated in the metastasis subgroup (FOXM1: $p=0.041$ ). In a multivariate analysis of FOXM1 and MDM2 in these 18 GEP-NEN samples, we found that MDM2 expression was the strongest covariate (beta coefficient $=0.530 ; p=0.013$ ).

To verify the data, we further stained paraffin-embedded tumor specimens of 18/23 patients (suppl. Table 2) with antibodies against MDM2, FOXM1, and p53. We were able to detect immunoreactivity against p53 (cytoplasmic or nuclear presence) only in $2 / 18$ cases. Those cases did express neither FOXM1 nor MDM2. MDM2 was strongly expressed in the nuclei of $8 / 18$ specimens. The signal was heterogeneous throughout the tumors, with a strong staining in the invasive peripheral regions.

We could further show a tendency of FOXM1 and MDM2 to be coexpressed ( $p=0.069)$. Whereas FOXM1 (nuclear) expression was significantly associated with dif-

Fig. 1. Expression of MDM2 in primary tumors and metastases: fresh frozen primary tumor and metastasis tissue derived from pancreatic and gastrointestinal (a) or gastrointestinal tumors only (b) were lysed and prospectively analyzed by Western blot. As internal control (int. ctrl.) pan-actin was used, all repeated measurements were normalized to a predefined sample. Densitometry analysis demonstrated a higher expression of MDM2 in the metastatic tissue of GEP-NEN in general $(p=0.036)$ and of gastrointestinal NEN $(p=0.049)$. c, d Representative FOXM1 and MDM2 Western blotting raw data of 4 analyzed patients. pNEC, pancreatic neuroendocrine carcinoma; iNEN, ileal neuroendocrine neoplasm; iNEC; ileal neuroendocrine carcinoma; MTS, metastasis; LN, lymph node; ferentiation (G1/G2 versus G3; $p=0.020$; Fisher exact test). Resulting from the low number of metastasis specimens, we were not able to verify the higher expression of MDM2 and FOXM1 in metastases.

\section{MDM2 Is Aberrantly Expressed, but the TP53 Status}

Does Not Reflect Clinically Relevant Genotypes in

Many Commonly Used GEP-NEN Cell Lines

Several studies have shown that TP53 is rarely mutated in GEP-NEN tissues, but extra gene copies of MDM2 or aberrant regulation of other upstream regulators might induce a functional loss of p53 [9]. To test the cellular response to MDM2 inhibition, a cell line with similar characteristics concerning p53 and MDM2 status was necessary. Therefore, we analyzed several common cell lines for aberrations in MDM2 gene copies and expression and for TP53 mutations.

Sequencing of the TP53 genomic region revealed homozygous deleterious mutations in 3 of the 4 cell lines (Fig. 2). The amino acid substitution that was detected in LCC-18 has been reported to destabilize the $\mathrm{H} 2$ helix of p53, leading to conformational changes within the DNAbinding domain [25]. In BON (Fig. 2a) and QGP-1 cells (Fig. 2a, b), TP53 mutations were found that lead to a premature stop codon and potentially an insufficient expression of the proteins. In BON cells this led to the disruption of the tetramerization motif, and in QGP-1 cells the mutation disrupts the DNA-binding domain as well as the tetramerization motif. Hence, we could not detect any p53 expression in QGP-1 by Western blot with the used antibodies, not even under nutlin-3a treatment and/or cytotoxic stress induction by cisplatin (data not shown). The FISH analysis did not show MDM2 amplification in BON and KRJ-I cells (Fig. 2c, d), but polyploidy of chromosome 17 in LCC-18 (Fig. 2e) and QGP-1 cells and additional copy numbers in QGP-1 cells (Fig. 2f).

PT, primary tumor. Biological replicates (biol. repl.) from different tumor areas were used if available, measured independently, and the median of all replicates was used in further statistical analysis. e, $\mathbf{f}$ Immunohistochemistry of MDM2 in FFPE material demonstrated a heterogeneous distribution throughout the tumor, a dominant staining in the tumor periphery, and a negative immunoreactivity in the surrounding tissue (e). The strong nuclear expression of MDM2 was found in 44\% of the analyzed specimens (f). $\mathbf{g}$, $\mathbf{h}$ Representative picture of a FOXM1-positive tumor (g) and picture of a case of weak p53 positivity (h). P53 could only be detected in the cytoplasm. i Positive control of p53 nuclear staining (breast cancer). Light microscopy. Magnification $\times 100$. 


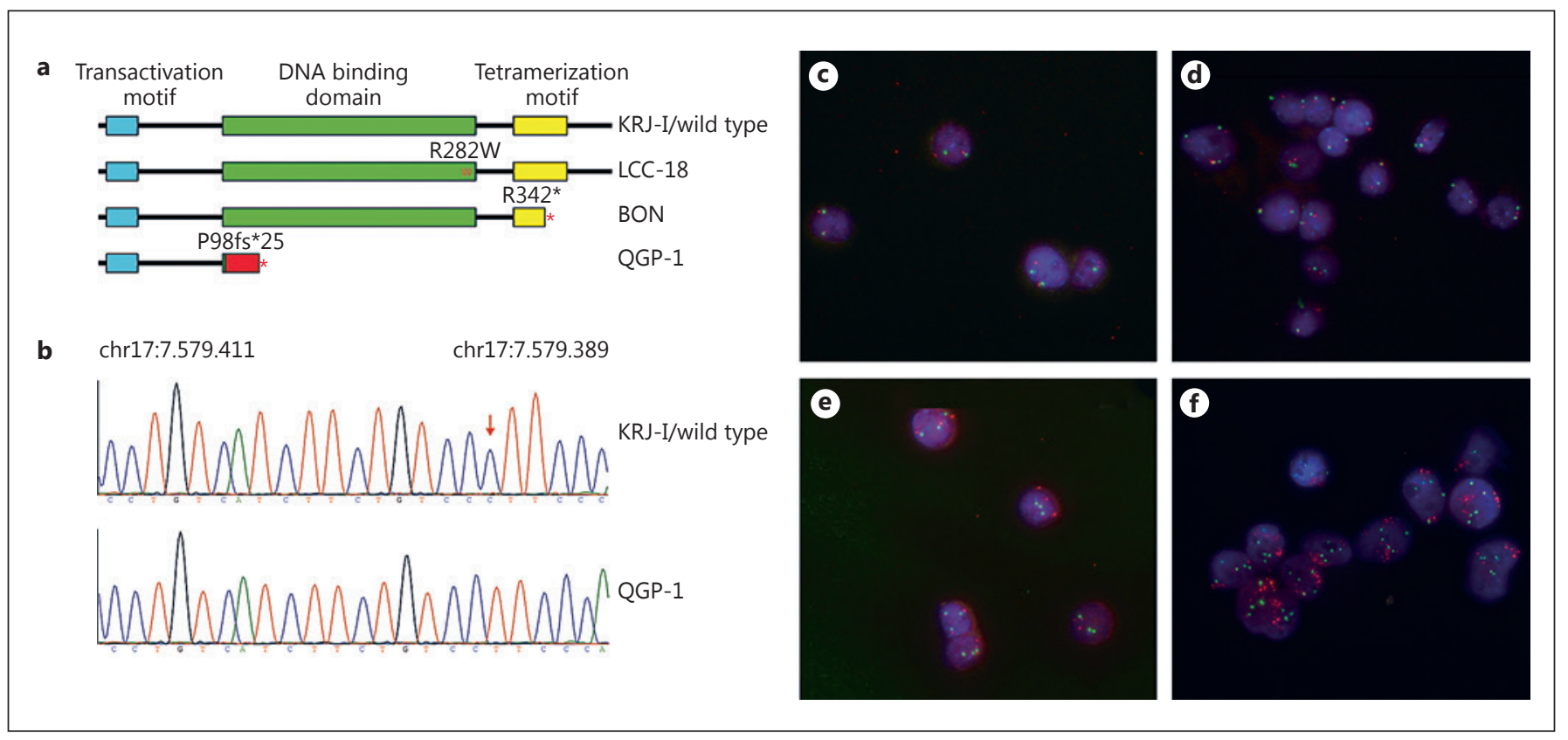

Fig. 2. Determination of the TP53 and the MDM2 status of GEPNEN cell lines: cell lines were analyzed by deep sequencing of the TP53 gene. a Schematic illustration of the resulting transcripts (major domains and site of mutation): sequencing revealed a mutation resulting in an amino acid substitution at position 282 (R282W) of TP53 in LCC-18 cells. In BON cells, a nonsense mutation at position $342\left(\mathrm{R} 342^{*}\right)$ led to the introduction of a premature stop codon. In QGP-1, a single base deletion in exon 4 was predicted but did not reach all quality criteria for variant calling. b Additional Sanger sequencing of the respective region confirmed

As TP53 wild-type expression is a key prerequisite of MDM2-p53 interaction inhibitory therapy approaches, we chose KRJ-I as research object and BON or QGP-1 as negative control.

\section{Small-Molecule MDM2-p53 Interaction Inhibitors}

Inhibit Proliferation in a TP53 Wild-Type Small

\section{Intestinal Neuroendocrine Cell Line}

In order to assess the MDM2-p53 interaction as therapeutic target in GEP-NEN, we treated a small intestinal (KRJ-I) and a control pancreatic neuroendocrine cell line (BON) with nutlin-3a (Fig. 3). Nutlins are reversible p53competitive inhibitors and inhibit the MDM2-p53 interaction by binding the special interacting binding pocket of MDM2, whereas other binding pockets remain active.

We could find a significant linear trend in BON cells after $72 \mathrm{~h}(p=0.0001)$, but with a high theoretical $\mathrm{IC}_{50}$ and a low maximum effect (Fig. 3a). BON cells are thus considered resistant to nutlin-3a, as expected. the deletion, resulting in a frame shift at position 98 of TP53 and a premature stop codon at position 122 (P98fs*25). Fluorescence in situ hybridization (FISH) analyses of the cell lines revealed a normal MDM2 copy number in BON (c) and KRJ-I (d) cells, whereas the LCC-18 (e) cells show a trisomic chromosome 17 and a resulting third copy of MDM2. The p53 deleterious cell line QGP-1 (f) was found to be pentasomic for chromosome 17 and with an MDM2 amplification cluster, resulting in 25 MDM2 fluorescent signals. Orange probe: $M D M 2$; green probe: chromosome enumeration probe (alpha satellite DNA).

In contrast, KRJ-I responded significantly and with a high maximum effect to the treatment with nutlin-3a (Fig. $3 \mathrm{~b} ; p<0.0001$ ). Here, we assessed relative $\mathrm{IC}_{50}$ values of approximately $1.3-1.6 \mu \mathrm{M}$.

We further treated KRJ-I and BON as well as pancreatic QGP-1 and colonic LCC-18 GEP-NEN cell lines with the racemic isoform of nutlin-3a, namely nutlin-3. We could show that only KRJ-I cells responded to nutlin-3 (online suppl. Fig. 1).

\section{Combined Treatment of KRJ-I Cells with Nutlin-3a}

Sensitizes to Cisplatin Chemotherapy

Enhanced effects of recovering a proapoptotic p53 function and induction of genotoxic stress are probable, and a sensitizing effect of a combined treatment has been demonstrated for several cancer entities [26-28]. Furthermore, nutlin-3a has been shown to inhibit FOXM1 activity and expression, which is usually activated after cisplatin treatment and contributes to platin-based che- 


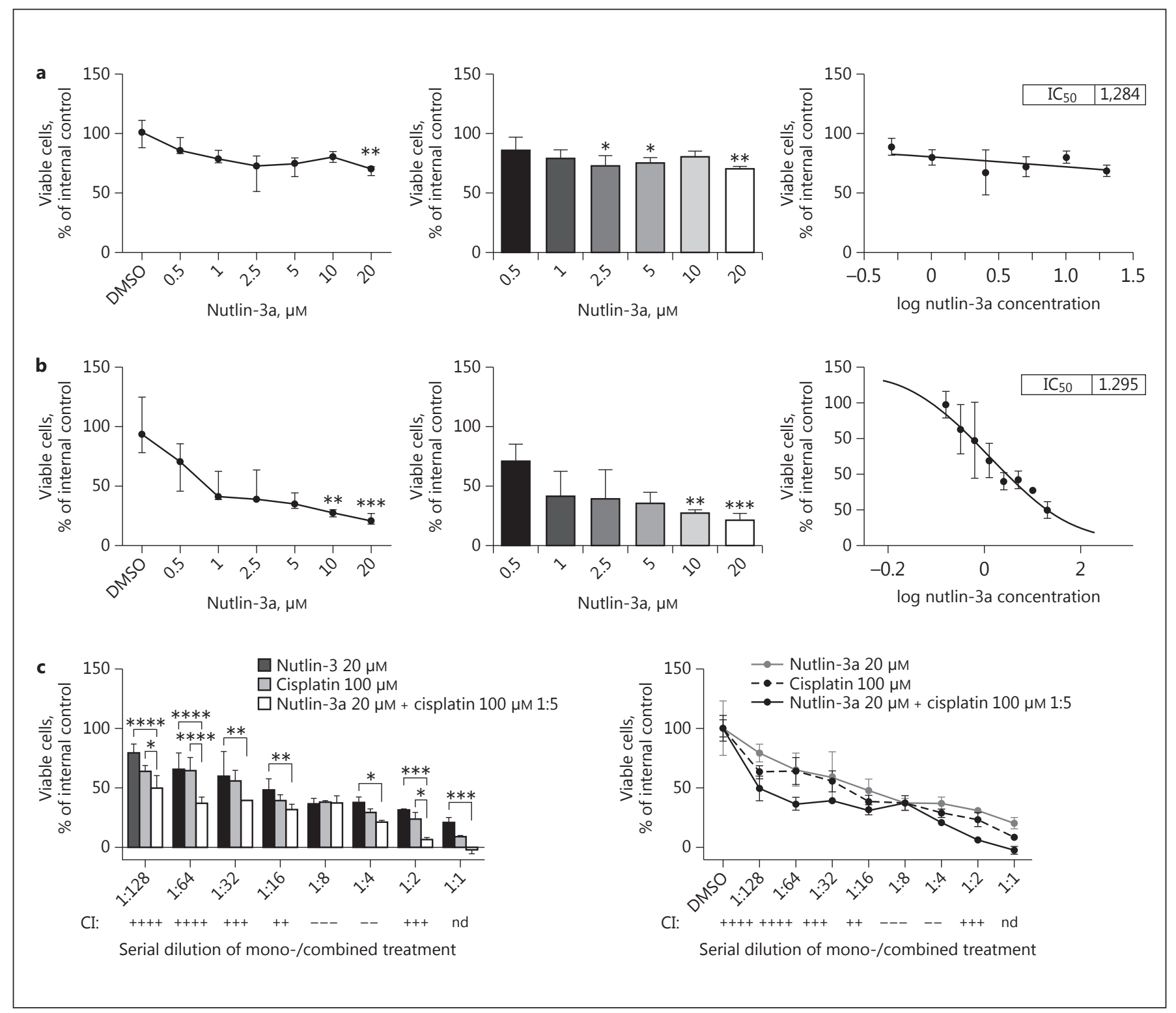

Fig. 3. Antiproliferative effects of the cis-imidazoline analog nutlin-3a in GEP-NEN cell lines in vitro: cells were treated in quintuplets with increasing concentrations of nutlin-3a for $72 \mathrm{~h}$ and analyzed with WST-1 proliferation assays. a BON cells responded only very weakly to nutlin-3a. The maximum effect resulted in a very high theoretical $\mathrm{IC}_{50}$ of $1,284 \mu \mathrm{M}$, indicating no relevant response. b Dose-response data of KRJ-I cells: proliferation was inhibited significantly after $72 \mathrm{~h}$ treatment with nutlin-3a (ANOVA; $p<$ 0.0001 ) with an $\mathrm{IC}_{50}$ of approximately $1.3 \mu \mathrm{M}$ (confidence interval:
0.95-1.76 $\mu \mathrm{M})$. c KRJ-I cells were treated with increasing concentrations of nutlin-3a (gray line), cisplatin (dashed black line), or a 1:5 combination of both substances (black line) for $72 \mathrm{~h}$. The dilution ranged from $780 \mathrm{nM}$ cisplatin $+156 \mathrm{nM}$ nutlin-3a to $100 \mu \mathrm{M}$ cisplatin $+20 \mu \mathrm{M}$ nutlin-3a. Combinatory effects were quantitated by the Chou-Talalay algorithm [23] and visualized as follows: strong synergism, ++++; synergism, +++; moderate synergism, ++ ; moderate antagonism, - -; antagonism, - - - ${ }^{*} p<0.05$, ${ }^{* *} p<0.01,{ }^{* * *} p<0.001,{ }^{* * * *} p<0.0001$; nd, not determined. motherapy resistance [29-31]. We therefore assessed the in vitro antiproliferative effect of nutlin-3a combined with cisplatin. We found a robust enhanced effect of combined nutlin-3a/cisplatin treatment with respect to each substance alone in KRJ-I cells (Fig. 3c). Here, the $\mathrm{IC}_{50}$ of cisplatin could be reduced by the factor of 3 (from 3.06 to $0.95 \mu \mathrm{M}$ ), whereas the $\mathrm{IC}_{50}$ of nutlin-3a was decreased approximately 8 -fold (from 1.293 to $0.1689 \mu \mathrm{M}$ ). 


\section{Nutlin-3a Induces Apoptosis in p53 Wild-Type}

GEP-NEN

To assess the detailed effect of nutlins on GEP-NEN cell lines, we performed mitotic index and JC-1 flow cytometry. Treatment of BON and QGP-1 cells with $5 \mu \mathrm{M}$ nutlin-3a did not affect the cell cycle, the mitotic index, or apoptosis (Fig. 4a, b). KRJ-I cells responded to the treatment by a significantly enhanced induction of apoptosis (indicated by the sub-G1 population) related to the controls (Fig. 4c, $p=0.0022$ ) and decreased mitosis. To further verify the sub-G1 effect as apoptosis, we performed a JC-1 apoptosis flow cytometry with $5 \mu \mathrm{M}$ nutlin3a to quantify changes in mitochondrial membrane potential indicating early apoptosis (Fig. 4d). The nonresponding control cell line QGP-1 did not alter their color pattern, whereas KRJ-I cells responded significantly to nutlin-3a treatment after 16 and 20 h, respectively. We could further demonstrate an enhanced proapoptotic effect of nutlin-3a combined with $10 \mu \mathrm{M}$ cisplatin compared to the nutlin-3a monotreatment. a

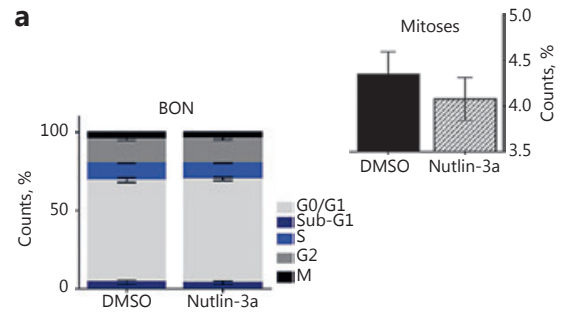

b

c

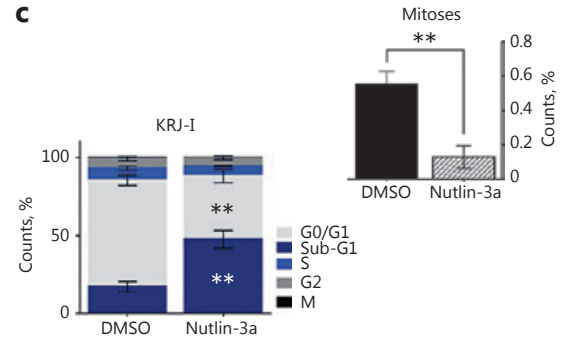

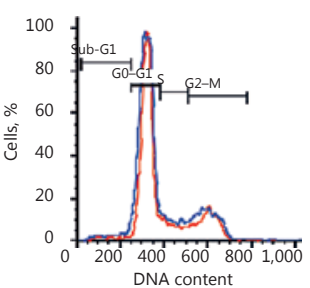

d
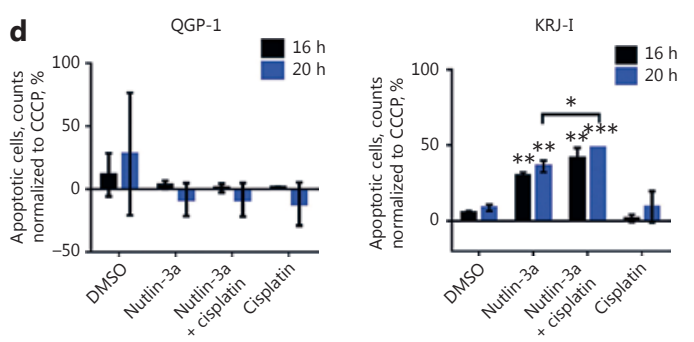

e
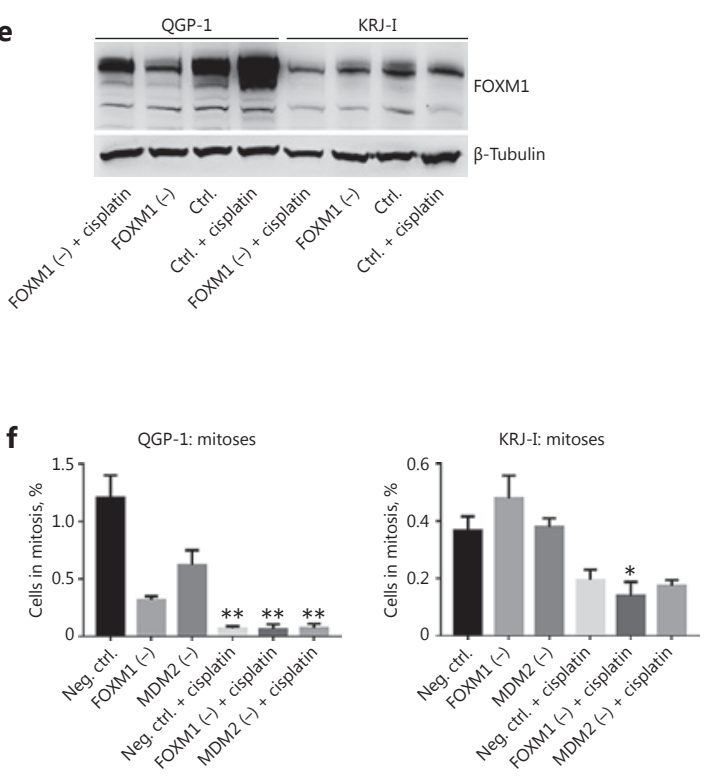

g

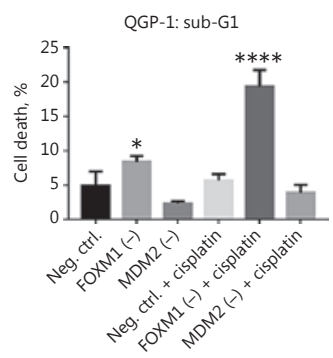

4

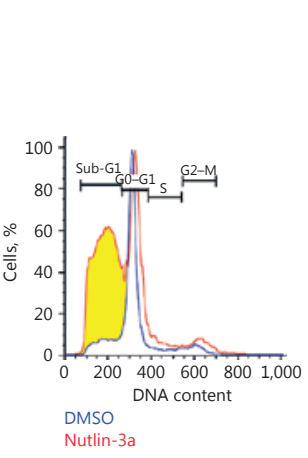

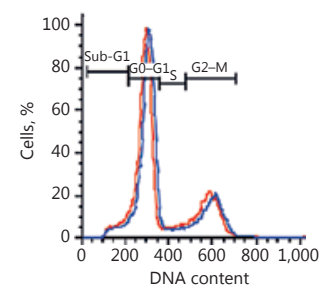

Nutlin-3

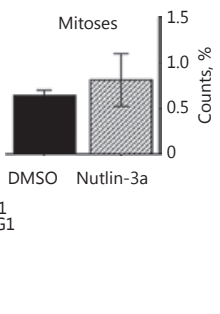


To address whether an antiproliferative effect of cisplatin can not only be enhanced by nutlin-3a, but also by reduction of MDM2 or FOXM1 expression, we treated MDM2 or FOXM1 knockdown QGP-1 and KRJ-I cells with cisplatin. We found a significantly reduced mitotic population after FOXM1 knockdown + cisplatin related to the controls in both cell lines, but these effects were only slightly stronger than the monotherapeutic effect of cisplatin alone (Fig. 4f). In QGP-1, the knockdown of FOXM1 alone induced a significant increase in the subG1 population, which indicated increased cell death. This effect was strongly enhanced by addition of cisplatin $(p<$ $0.0001)$.

\section{Nutlin-3a Triggers the Nuclear Translocation of p53} and Thereby Enables p53 Downstream Effects

After ubiquitination by its negative regulator MDM2, p53 is exported from the nucleus and undergoes proteasomal degradation. In untreated KRJ-I cells, p53 is located in the cytoplasm (Fig. 5a), indicating MDM2 activity and an inactive transcription factor function. Those cells further show a predominant localization of MDM2 in the nucleus and a strong cytoplasmic FOXM1 staining (Fig. 5c). After treatment of the cells with nutlin-3a, p53 translocates into the nucleus (Fig. 5b) and thereby triggers the de novo expression of its negative feedback regulator MDM2, which is a typical indicator for wild-type p53 activity and is indicated by an increased perinuclear immunoreactivity (and thus localization near the endoplasmic reticulum) of MDM2 (Fig. 5d). Furthermore, nutlin-3a-treated cells lose their FOXM1 immunoreactivity (Fig. 5d); as cells with nuclear (active) p53 localization do not express the p53 target FOXM1 and cells with cytoplasmic (inactive) p53 retain FOXM1 expression (Fig. 5e). These results show the strong dependency of FOXM1 expression from p53 transcription factor activity.

\section{Nutlin-3a Reinduces Wild-Type p53 Signaling, Alters Gene Expression Patterns in Cancer-Related Canonical Pathways and Downregulates DNA Damage Repair Genes}

To examine the inductive effect of nutlin-3a concerning a wild-type p53 response, we performed a multiplex gene expression analysis using the PanCancer pathway nCounter ${ }^{\circledR}$ panel by Nanostring ${ }^{\circledR}$ technologies [32] of KRJ-I cells treated with $5 \mu \mathrm{M}$ nutlin-3a versus DMSO for $60 \mathrm{~h}$ and further verified the expression of selected proteins by Western blot. We found considerable changes in the gene expression pattern after nutlin-3a treatment (Fig. 6a). In untreated and DMSO-treated cells, MDM2 was one of the leading edge transcripts of the analyzed gene panel, along with $B 2 M\left(\beta_{2}\right.$-microglobulin), transcripts of histone $\mathrm{H} 3$ (H3F3C, HIST1H3B, HIST1H3H),

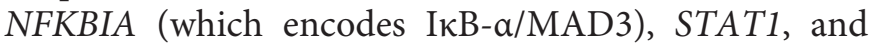
genes involved in ubiquitin homeostasis (SKP1, UBB, RPS27A) (Fig. 6b; for a complete listing of all 730 genes refer to nanostring.com).

After treatment with nutlin-3a, we found 212 of 730 transcripts with significantly altered differential expres-
Fig. 4. Treatment effects of nutlin-3a in vitro. BON (a), QGP-1 (b), and KRJ-I (c) cells were treated with $5 \mu \mathrm{M}$ nutlin-3a or DMSO for $72 \mathrm{~h}$ and analyzed by mitotic index flow cytometry. The p53 wildtype cell line KRJ-I displayed a significant increase in the sub-G1 population of the treatment group $(p=0.0022)$ after $72 \mathrm{~h}$, indicating the induction of apoptosis. Further, the mitotic population, indicated by a phospho-(serine 10) histone $\mathrm{H} 3$ immunoreactivity, was significantly decreased $(p=0.0022)$. d Changes in the membrane potential of the mitochondria were analyzed by JC-1 staining after 16 and $20 \mathrm{~h}$ of treatment with nutlin-3a in order to detect early apoptosis induction in KRJ-I and QGP-1 cells. Results were normalized to untreated and carbonylcyanide $m$-chlorophenylhydrazone (CCCP, a mitochondrial membrane depolarizer) controls. In KRJ-I cells, significant induction of apoptosis could be shown after 16 and $20 \mathrm{~h}(p=0.0064$ and $p=0.0009)$ of $5 \mu \mathrm{M}$ nutlin3 a treatment with respect to the DMSO controls. The apoptosis inductive effect of nutlin-3a combined with cisplatin was further significantly enhanced after $20 \mathrm{~h}$ in relation to nutlin-3a alone $(p=0.0191)$. e FOXM1 expression after knockdown of FOXM1 with and without cisplatin addition. Blots were cropped to im- prove conciseness. f, g QGP-1 and KRJ-I cells were transfected with 30-50 nM alternative siRNAs against FOXM1, MDM2, and control siRNA for $24 \mathrm{~h}$. Cells were subsequently treated with $5 \mu \mathrm{M}$ cisplatin or saline control for $72 \mathrm{~h}$. Mitotic index (phospho-histone H3/PI staining) flow cytometry was performed. In QGP-1 cells, mitosis was reduced by the influence of cisplatin, rather than by knockdown of FOXM1 or MDM2, as also the cisplatin-treated control showed a significant decrease in mitotic cells. Here, the knockdown of FOXM1 significantly induced cell death, indicated by an increase in the sub-G1 population. The addition of cisplatin to FOXM1 knockdown QGP-1 cells further enhanced this effect $(p<0.0001)$. Furthermore, the sub-G1 population was larger than the sub-G1 population of the positive cell death control (data not shown; $p=0.0120$ ). In contrast, KRJ-I cells showed only a significant response after FOXM1 knockdown + cisplatin combination. Here, the additional knockdown of FOXM1 or MDM2 did not enhance the cytotoxic effect of cisplatin (indicated by sub-G1). Representative experiments shown. ${ }^{*} p<0.05,{ }^{* *} p<0.01$, ${ }^{* * *} p<$ $0.001, * * * * p<0.0001$, related to negative control (neg. ctrl.). 
p53
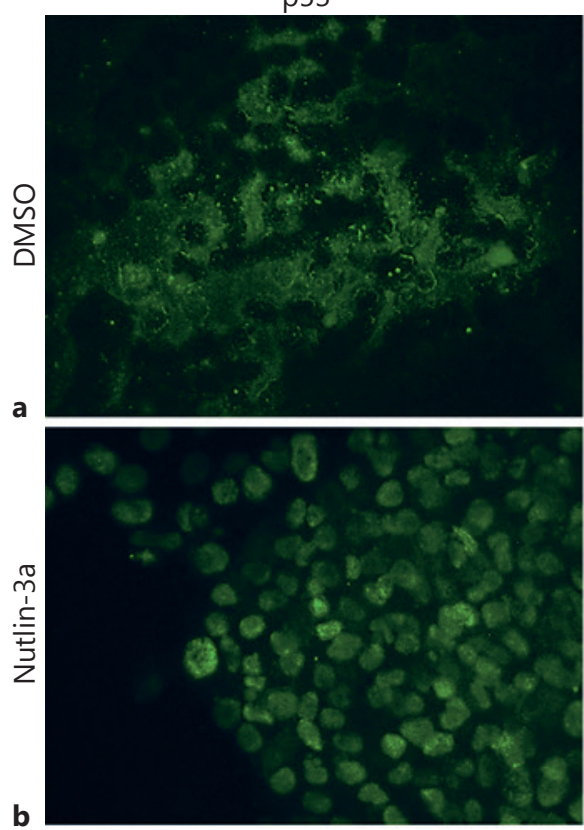

MDM2

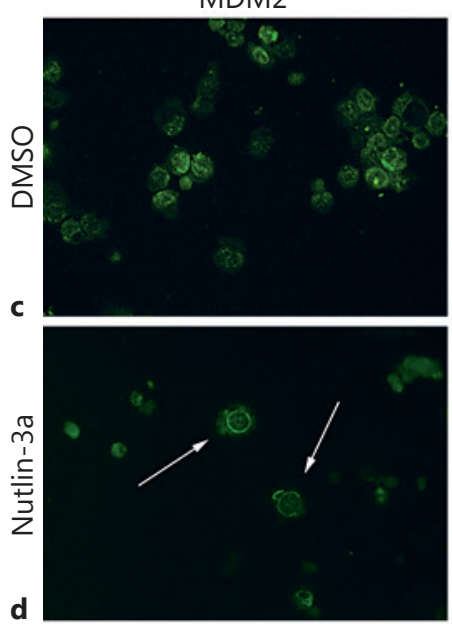

p53

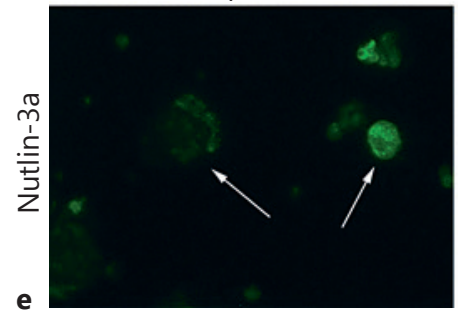

p53 + DAPI
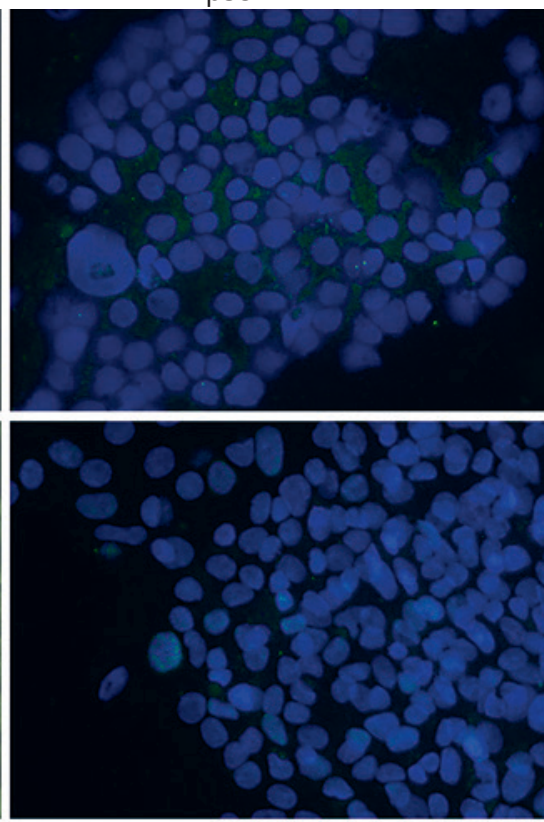

FOXM1

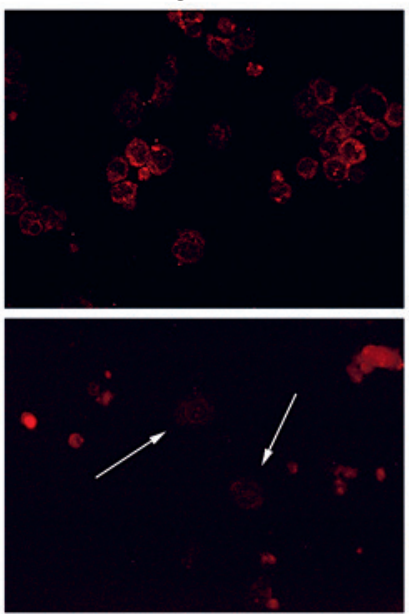

FOXM1

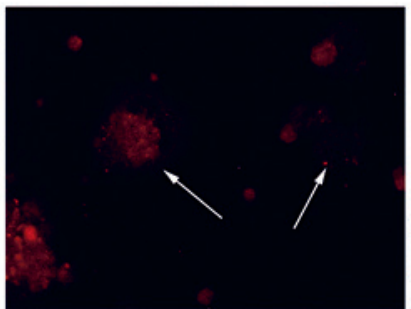

Merge + DAPI
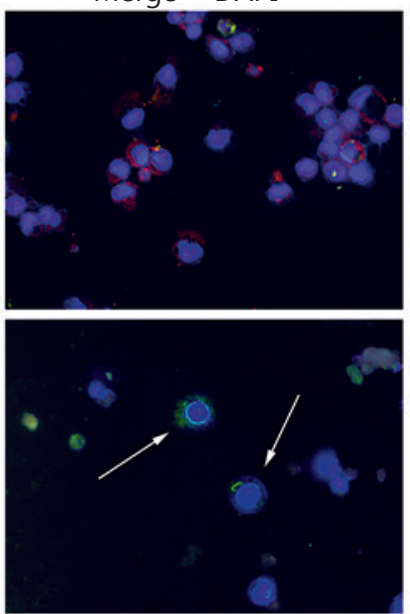

Merge + DAPI

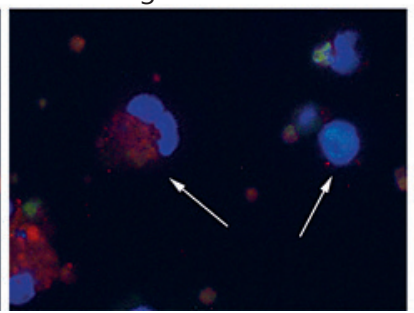

Morphology
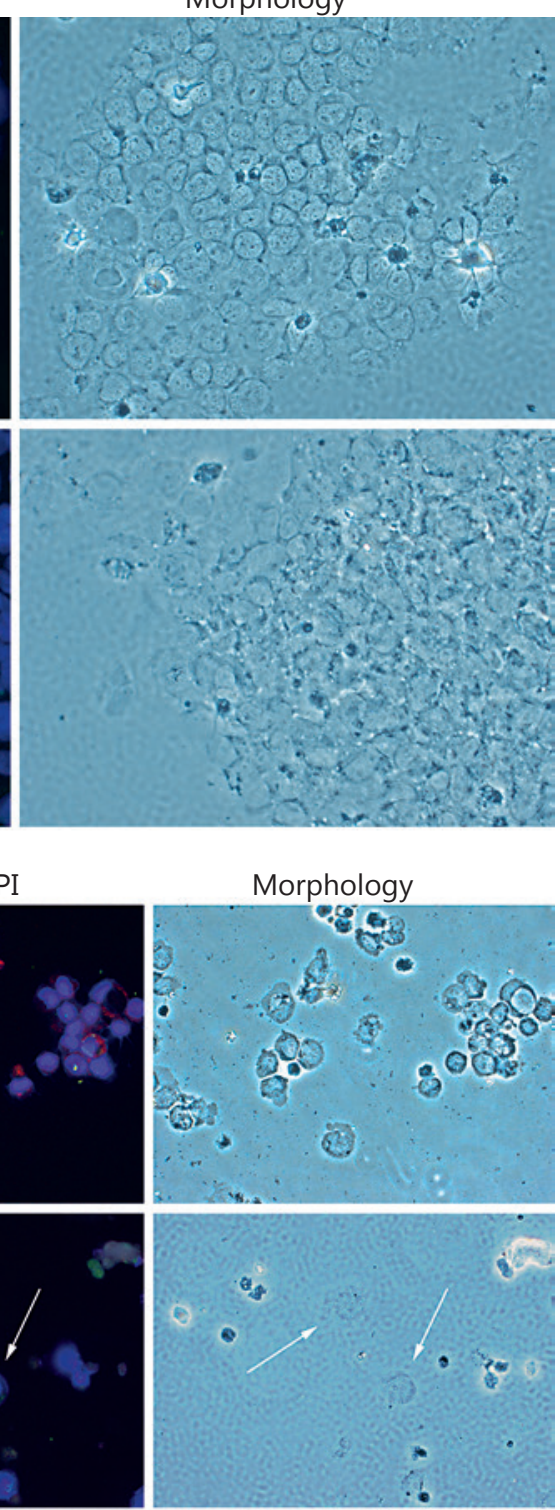

Morphology

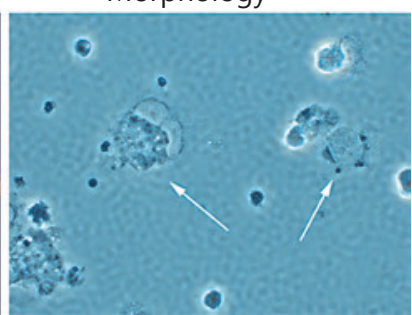


sion. The 42 statistically most significant differentially expressed p53 target genes with a differential expression $> \pm 1.5$-fold can be found in Figure 7. All transcripts listed in this table are direct transcriptional targets with known experimentally or in silico validated response elements for p53 family proteins (refer to p53FamTaG database [33]). All significantly altered transcripts can be found in online suppl. Table 3.

The main effect of nutlin-3a affected the cell cycle regulation, where a large number of positive regulators were downregulated (online suppl. Fig. 2). In order to categorize the differentially expressed genes by biological function we performed a PANTHER pathway GO overrepresentation analysis of all differentially expressed genes. The analysis demonstrated the overrepresentation of genes associated with DNA recombination $(+13.33$-fold, $p=0.000307)$, DNA repair $(+11.85$-fold, $p=6.14 \mathrm{E}-14)$, DNA replication $(+8.77-$ fold, $p=3.10 \mathrm{E}-7)$, cell proliferation (+7.42-fold, $p=9.82 \mathrm{E}-5)$, regulation of cell cycle (+6.24-fold, $p=0.0379)$, cytokine-mediated signaling pathway $(+6.1$-fold, $p=0.000636)$, mitosis $(+5.22$-fold, $p=6.68 \mathrm{E}-7)$, and further $\mathrm{GO}$ annotations.

Most notably to prove the 53 activity in principle, the major direct targets CDKN1A (which encodes p21) und p53 regulators such as $M D M 2$ and LIF (which contribute to autoregulatory loops [34]) were upregulated. This indicates an intact primary p53 response (Fig. 6-8; online suppl. Table 3).

The altered expression of p53 and of the p53 targets FOXM1, MDM2, and p21 after nutlin, cisplatin, and combined treatment was confirmed by Western blotting. We could detect significant changes in the protein abundance of p21, MDM2, FOXM1, and p53 in KRJ-I cells after nutlin-3a and combined treatment (Fig. 8).

We could further verify the p53 stabilization by serine-20 phosphorylation, induction of BAX, decreased expression of FOXM1 and DNA damage repair proteins, such as SKP2, RAD51, and FEN1, and an associated increased DNA damage stress, indicated by histone H2AX phosphorylation in KRJ-I (Fig. 8a, b).

Fig. 5. Subcellular changes in protein localization after nutlin-3a treatment: KRJ-I cells were treated with nutlin-3a or DMSO control for $72 \mathrm{~h}$ and costained for immunofluorescence microscopy with antibodies against p53, MDM2 and FOXM1. a In control treated cells, p53 was predominantly localized in the cytoplasm, as it was also detected in the immunohistochemistry specimens. b p53 translocated into the nuclei after treatment with nutlin-3a, indicating an increase in p53 transcription factor activity. c Control treated cells show a strong nuclear expression of MDM2 and
The combined treatment of nutlin and cisplatin (Fig. 8c, d) did not increase proapoptotic signaling after $72 \mathrm{~h}$ (e.g. by induction of caspase 9 or PARP cleavage). We further repeatedly observed a lower abundance of $\mathrm{p} 21$ after combination therapy in relation to nutlin monotherapy indicating a shift in the resulting cell cycle arrest mechanisms. Here, we found the expression of the mitotic regulator FOXM1 and SKP2 (which regulates p27 expression via the Skp-Cullin-F-box complex) reduced in KRJ-I cells (Fig. 8c). FOXM1 and its target SKP2 were not affected in the p53-deficient cell line QGP-1 (Fig. 8d).

After nutlin-3a treatment of p53 wild-type KRJ-I cells, we found 2 changes in FOXM1 regulation: a reduced phosphorylation and a downregulation of all isoforms of FOXM1 (Fig. 9). The phosphorylation of the FOXM1 regulatory serine-35 in the autorepressor domain is required for the activation of FOXM1 [35]. Its hypophosphorylation indicates less FOXM1 activity.

\section{MDM2 Is Coexpressed with FOXM1 and Is Able to Regulate FOXM1 Independently from p53 in \\ GEP-NEN Cell Lines}

The crucial oncogene and p53 target FOXM1 [16] is strongly expressed in higher proliferative intestinal NEN tumor specimens and was associated with metastasis [17]. Strong expression of negative p53 regulators (MDM2, MDM4, and WIP1) has also been demonstrated to go along with metastatic progression in pancreatic NEN. In the current study, we have shown an association of high MDM2 expression with occurrence of distant metastases in GEP-NEN.

By immunofluorescent costaining of MDM2 and FOXM1 in ileal KRJ-I (Fig. 10a, b) and pancreatic BON (Fig. 10c, d) cell lines, we could demonstrate that untreated cells with a strong nuclear localization of MDM2 also show a strong expression of FOXM1. Interestingly, a large number of double-positive cells were multinucleated (Fig. 10a, c), indicating an enhanced mitotic instability in these cells. Mitotic instability can be induced by aberrant expression of mitotic kinases [36] such as

predominantly cytoplasmic localization of FOXM1. d After treatment with nutlin-3a, the MDM2 immunoreactivity shifted to the perinuclear space indicating a de novo MDM2 synthesis as result of increased p53 activity. In those cells, FOXM1 expression is lost due to p53 activation. e Costaining of p53 and FOXM1 after nutlin-3a treatment demonstrates that cells that acquire nuclear translocation of p53 undergo loss of FOXM1 expression. Cells that retain cytoplasmic p53 expression also retain their FOXM1 immunoreactivity. Fluorescence microscopy. Magnification $\times 400$. 

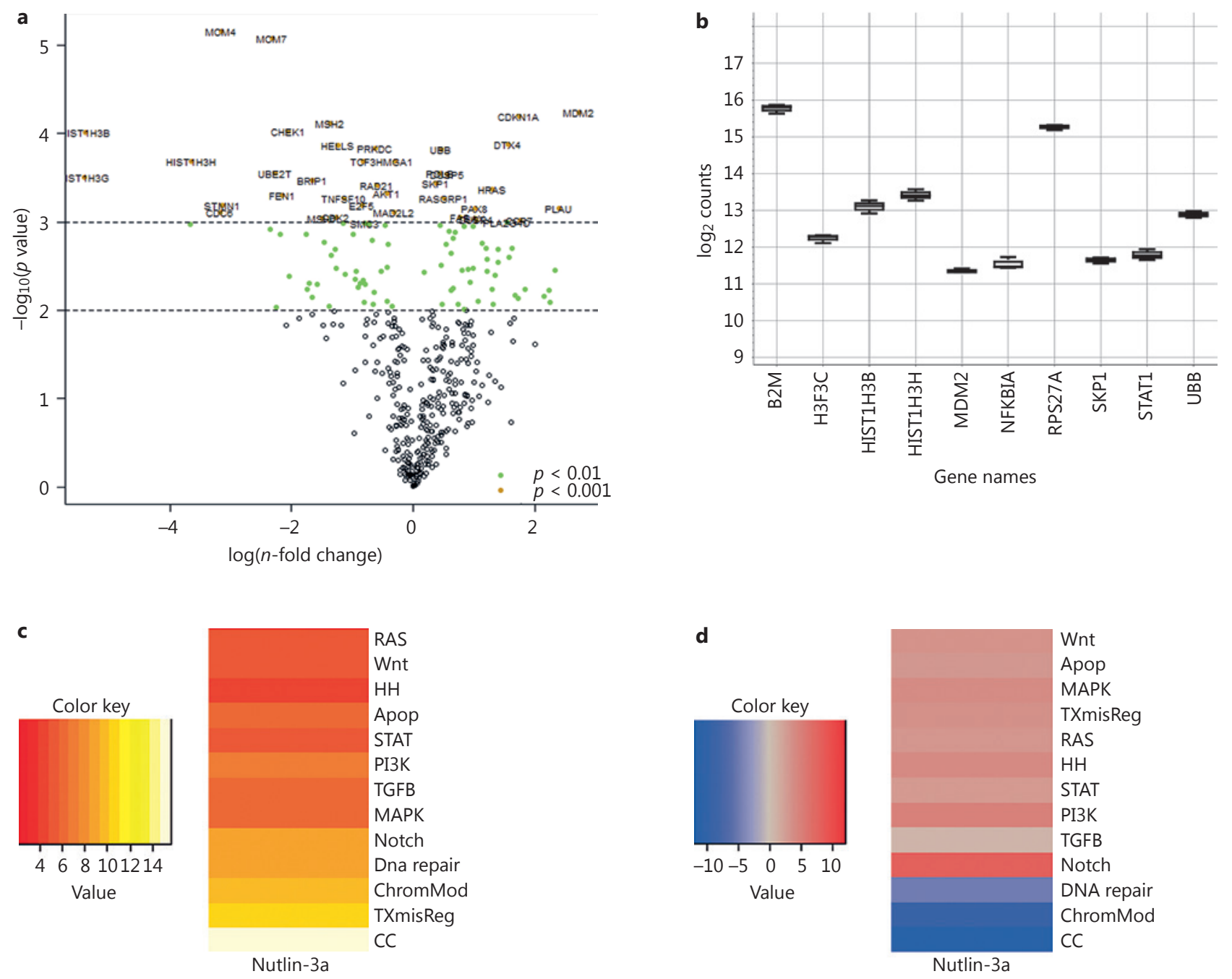

Fig. 6. Altered gene expression after $60 \mathrm{~h}$ of treatment with nutlin$3 a$ a The volcano plot of altered genes displays each gene's $-\log _{10}(p$ value) and $\log _{2}$ ( $n$-fold change) after treatment compared to DMSO controls. Highly statistically significant genes can be found at the top of the plot, and highly differentially expressed genes fall to either side. Horizontal lines indicate thresholds for $p=0.01$ and $p=$ 0.001 . After nutlin-3a treatment, it shows several strongly altered genes including genes encoding histone $\mathrm{H} 3$ proteins (-42-fold), cyclin A2 (-12.6-fold), MCM4 (-8.9-fold), MCM7 (-5.0-fold), MSH2 (-2.6-fold), p21 (+3.3-fold), and MDM2 (+6.6-fold). b MDM2 was found among the 10 most strongly expressed genes (of the PanCancer ${ }^{\circledR}$ panel) even in untreated conditions in KRJ-I cells. c, $\mathbf{d}$ Heat maps of affected pathways after nutlin-3a treatment (differential expression versus DMSO controls). c The heat map displays each group's global significance scores and thus measures the extent of differential expression of a pathway's genes with the treatment, ignoring whether each gene is up- or downregulated.
Yellow denotes pathways whose genes exhibit extensive differential expression after treatment, red denotes pathways with less differential expression. Nutlin-3a strongly altered the expression of genes involved in cell cycle regulation, transcriptional deregulation, chromatin modification, DNA repair, and notch. $\mathbf{d}$ The heat map displays each treatment's directed global significance scores. Directed global significance statistics measure the extent to which a pathway's genes are up- or downregulated after treatment. Red denotes pathways whose genes exhibit extensive overexpression with the covariate, blue denotes pathways with extensive underexpression. The blue regions indicate that genes of the most strongly affected pathways, e.g. cell cycle regulation, chromatin modification, and DNA repair, were predominantly underexpressed after treatment. Only notch signaling had a positive significant score indicating upregulation. Apop, apoptosis; TXmisReg, transcriptional misregulation; CC, cell cycle; ChromMod, chromatin modifications; $\mathrm{HH}$, hedgehog. 
Fig. 7. Relevant p53 targets after $60 \mathrm{~h}$ of nutlin-3a treatment of KRJ-I cells: altered p53 target genes with the lowest $p$ values $(p<0.005)$ and a differential expression $> \pm 1.5$-fold (dark: FDR $\leq 0.05$ ). Error bars indicate upper and lower confidence intervals. Negative $\log _{2}(n$-fold change) denotes repression while a positive $\log _{2}(n$-fold change) indicates induction. FDR, false discovery rate.

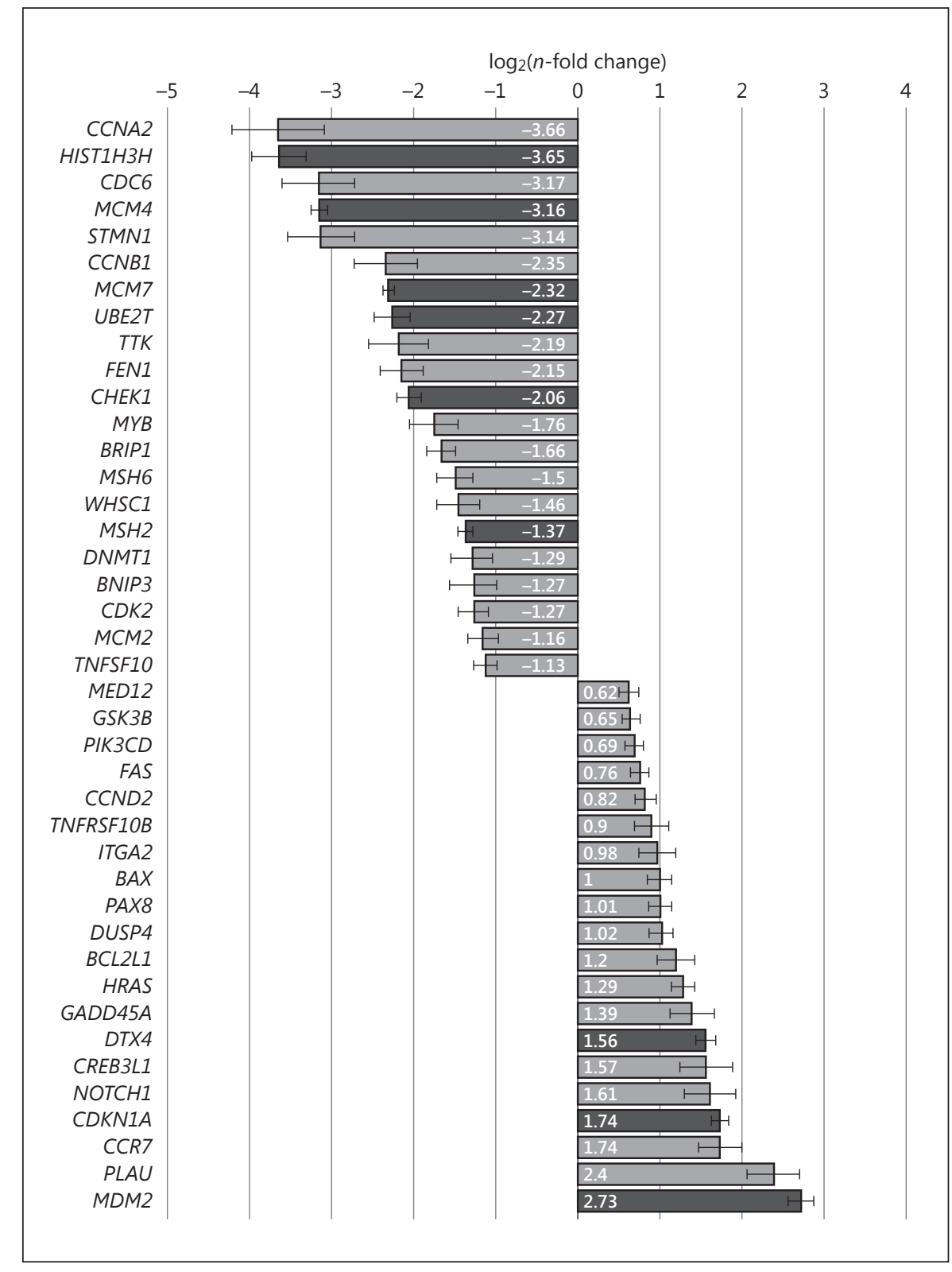

aurora A, which is a p53 and FOXM1 target. Interestingly, the p53 wild-type cell line KRJ-I only showed cytoplasmic FOXM1 localization. Therefore, there is also evidence that the sequestration of the transcription factor FOXM1 to the cytoplasm might have a further regulatory effect dependent on the actual TP53 genotype.

FOXM1 expression downstream of MDM2 seems to be dependent on and independent of $\mathrm{p} 53$. In contrast to the wild-type cell line, the treatment of the p53-defective QGP-1 cell line did not induce a 553 response after nutlin-3a, indicated by no upregulation of MDM2 and p21
(Fig. 11a). Nevertheless, the MDM2 knockdown in this p53-defective cell line decreased the FOXM1 expression and the expression of its targets aurora A and SKP2. Therefore, it must be independent from p53.

In the p53 wild-type cell line, nutlin-3a induced a de novo protein expression of MDM2 and p21, which was resulting from $\mathrm{p} 53$ activation. This effect was able to neutralize the effect of the MDM2 siRNA, as siRNA against MDM2 reduces MDM2 expression, but nutlin-3a and nutlin-3a combined with MDM2 siRNA induced MDM2 expression in a p53-dependent manner. Nevertheless, the 
Fig. 8. Effect of nutlin-3a and cisplatin combined therapy in p53 wild-type and p53-deficient GEP-NEN cell lines (Western blot). a, b After 72 h of $5 \mu \mathrm{M}$ nutlin-3a monotherapy of wild-type KRJ-I cells, the increased expression of direct p53 targets, such as $\mathrm{p} 21, \mathrm{MDM} 2, \mathrm{BAX}$ and reduction of FOXM1 could be verified. No increase in markers of extrinsic apoptosis induction pathways, such as FADD or TRADD, and only slight changes in caspase 8 cleavage could be shown. Nevertheless, DNA damage-related genes, such as FEN1, SKP2 or RAD51, were downregulated after nutlin3a treatment resulting in increased DNA damage stress, indicated by hyperphosphorylation of histone H2AX. Furthermore, a higher abundance of cleaved BID and BAX was visible. c, d The 72-h combination treatment of KRJ-I cells with nutlin3a plus cisplatin did not increase PARP cleavage. Furthermore, the expression of p21, p53, and MDM2 slightly decreased after combined treatment compared to monotherapy. The expression of FOXM1 was highly affected after combined therapy. QGP-1 cells do not show changes in FOXM1 or SKP2 expression and no induction of p53. Nevertheless, there is an unspecific induction of $\mathrm{p} 21$ after combined treatment. As the p53 detection of $\mathbf{c}$ and $\mathbf{d}$ was performed on identical blots, false-negative results concerning the p53 expression in QGP-1 cells can be excluded. Blots were cropped to improve conciseness. a

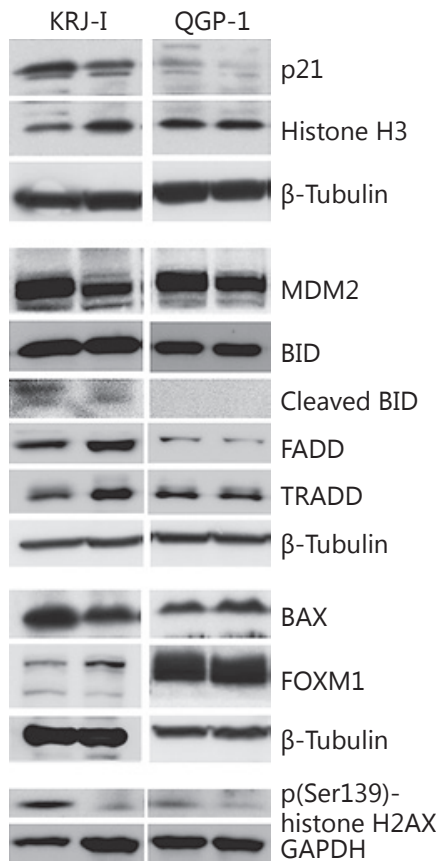

b

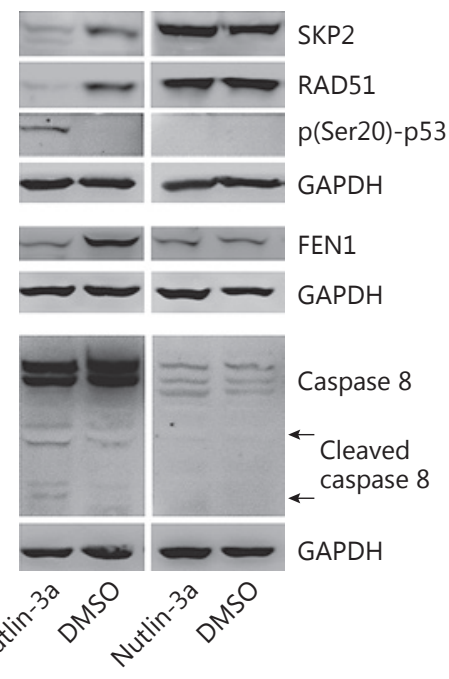

C
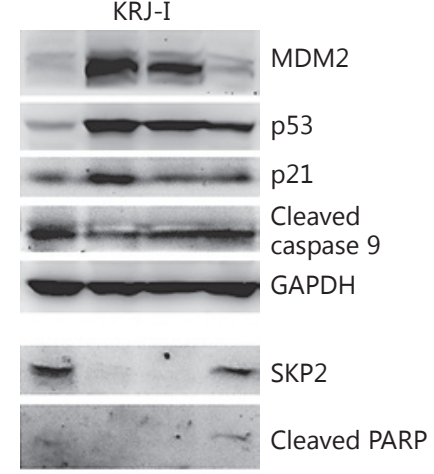

FOXM1
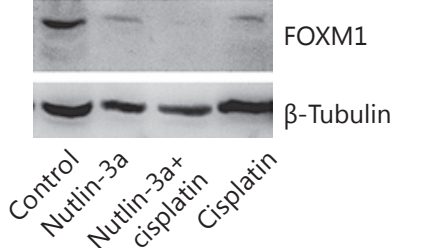

d
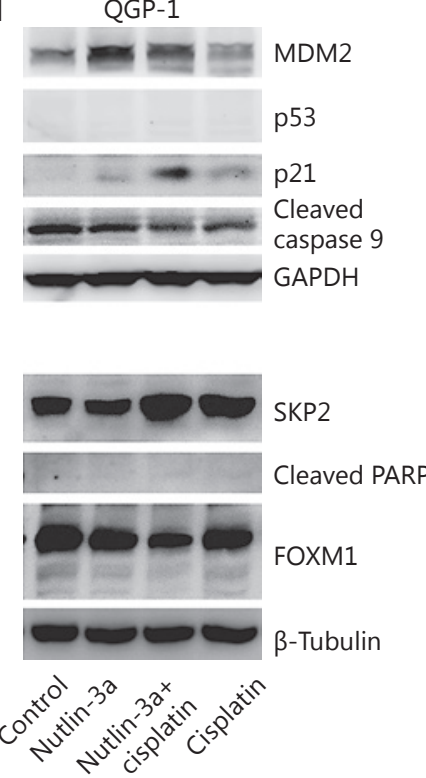

FOXM1 expression is decreased after knockdown of MDM2 (Fig. 11b) and is further reduced after p53 activation via nutlin-3a and thus is predominantly p53 dependent here.

Summarizing, nutlin-3a upregulates MDM2 via a p53MDM2 feedback loop in wild-type p53 KRJ-I cells, but lowers the FOXM1 activity p53-dependently. In p53-mutant GEP-NEN cells, nutlin-3a does not affect p53 activ- ity and MDM2 or FOXM1 expression, but MDM2 knockdown reduces FOXM1 protein abundance and is thus not mediated by $\mathrm{p} 53$.

\section{Treatment with Nutlin-3a Decreases Tumor Growth} in a Xenograft Mouse Model in vivo

KRJ-I cells were xenotransplanted subcutaneously in female NOG mice. Eight mice per group were treated with
16

Neuroendocrinology 2018;107:1-23 DOI: $10.1159 / 000481506$
Briest et al. 
Fig. 9. Regulation of FOXM1 by nutlin-3a. a Western blotting demonstrated a reduced serine-35 phosphorylation of FOXM1 after 72-h nutlin-3a treatment of KRJ-I cells, indicating a reduced activity. b Real-time PCR analysis of the FOXM1 expression after $72 \mathrm{~h}$ of $5 \mu \mathrm{M}$ nutlin-3a treatment showed that nutlin-3a downregulated all FOXM1 isoforms. Blots were cropped to improve conciseness.

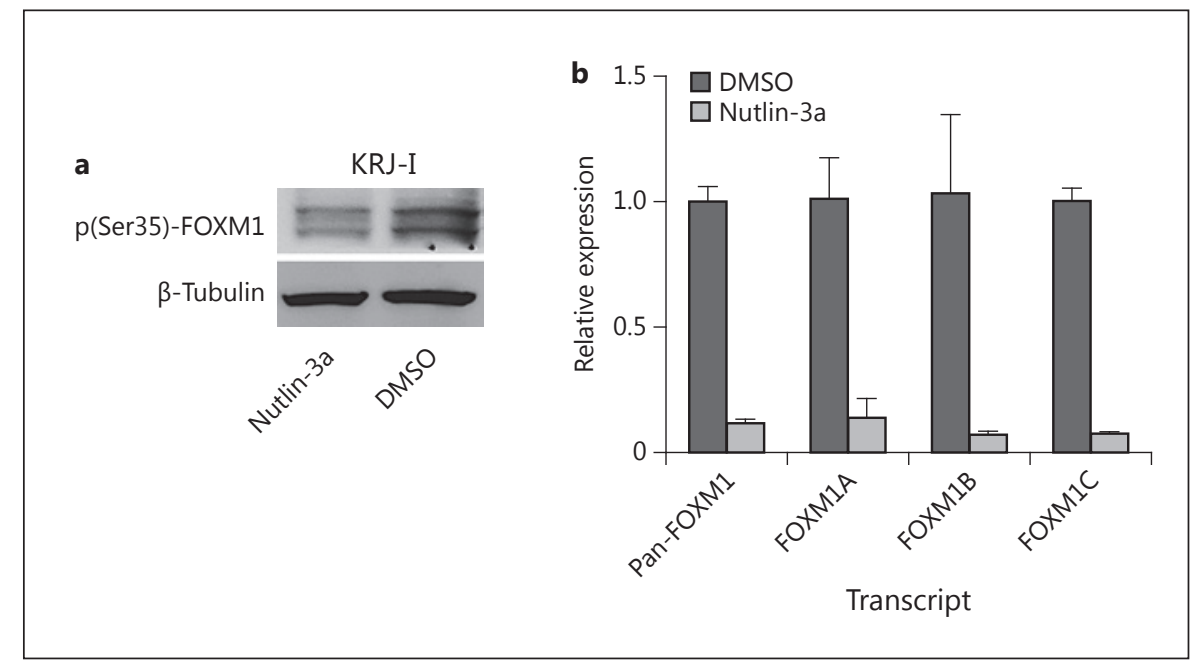

nutlin-3a or vehicle control, respectively. The tumor growth was significantly decelerated in the nutlin-3a treatment group after 49 days $(p<0.0001)$. Nutlin-3a therefore decreased tumor growth of KRJ-I cells in vivo (Fig. 12).

\section{Discussion}

In this study, we show detailed mechanisms of treating wild-type TP53 intestinal NEN with an MDM2 inhibitor in vitro and in vivo. Novel MDM2 targeting therapies are under investigation and might be suitable for this subset of tumors according to our study. The small-molecule inhibitor and cis-imidazoline analog nutlin-3a was used as a well-known model of MDM2-p53 interaction antagonists, and it was more effective than the commonly used therapeutic alternative everolimus in the p53 wild-type midgut cell line in vitro (online suppl. Fig. 1). Everolimus has recently been approved for progressive, nonfunctional intestinal NEN and has been applied for progressive pancreatic NEN since 2012. In a xenograft model, we could further demonstrate that nutlin-3a monotherapy decreased tumor growth in vivo.

The role of the p53 network in the development and progression of GEP-NEN is still poorly understood. The key players have already been characterized, and the most important finding was that TP53 is rarely mutated, but the pathway is deregulated (reviewed in Briest and Grabowski [14]). According to the upcoming WHO 2017 classification, TP53 mutation is a feature of poorly differentiated neuroendocrine carcinomas, and mutation analysis is recommended for distinction between G3 NET and G3 neuroendocrine carcinomas. In turn, p53 is consid- ered wild type in the well-differentiated G1-G3 NET and p53 recovery therapy might be promising in these tumors.

Most notably, we found nutlin-3a therapy supportive of cisplatin treatment, especially in the low doses, which might provide a rationale for a personalized treatment strategy of GEP-NEN patients with higher proliferative tumors that retained wild-type p53. As cisplatin is not effective in GEP-NEN in general, it is not used in the clinical context. It is further only slightly effective in GEP-NEN cell lines and therefore a good model to analyze the chemosensitizing effect of medical p53 reactivation. The possible reasons why platin-based chemotherapy is ineffective in GEP-NEN treatment are still not known. Reduced accumulation or enhanced efflux, inactivation by thiolcontaining molecules, deficiency in DNA damage sensing proteins (such as MMR), impaired induction of apoptosis or enhanced damage response might be possible mechanisms in general [37]. In GEP-NEN, a probable resistance mechanism is an activated DNA repair (i.e., by inactivation of p53 and thus activation of FOXM1 and its downstream DNA repair-related genes) and a slow proliferation rate (and thus an extended time for repair). In KRJ-I cells, p53 is not impaired by mutation, but, as shown by Hu et al. [9] and in this study, respectively, MDM2 is overactive. In KRJ-I cells it is predominant in the nucleus of this cell line, leading to p53 ubiquitination and nuclear export and thus a derepression of the FOXM1 gene locus. Because of the intense AKT signaling in NEN (combined with the clinically frequent MDM2 gene amplification), the MDM2 activity rises and sustained proliferation or tumorigenesis are possible consequences. Referring to data from other cancer entities, overexpressed MDM2 has 

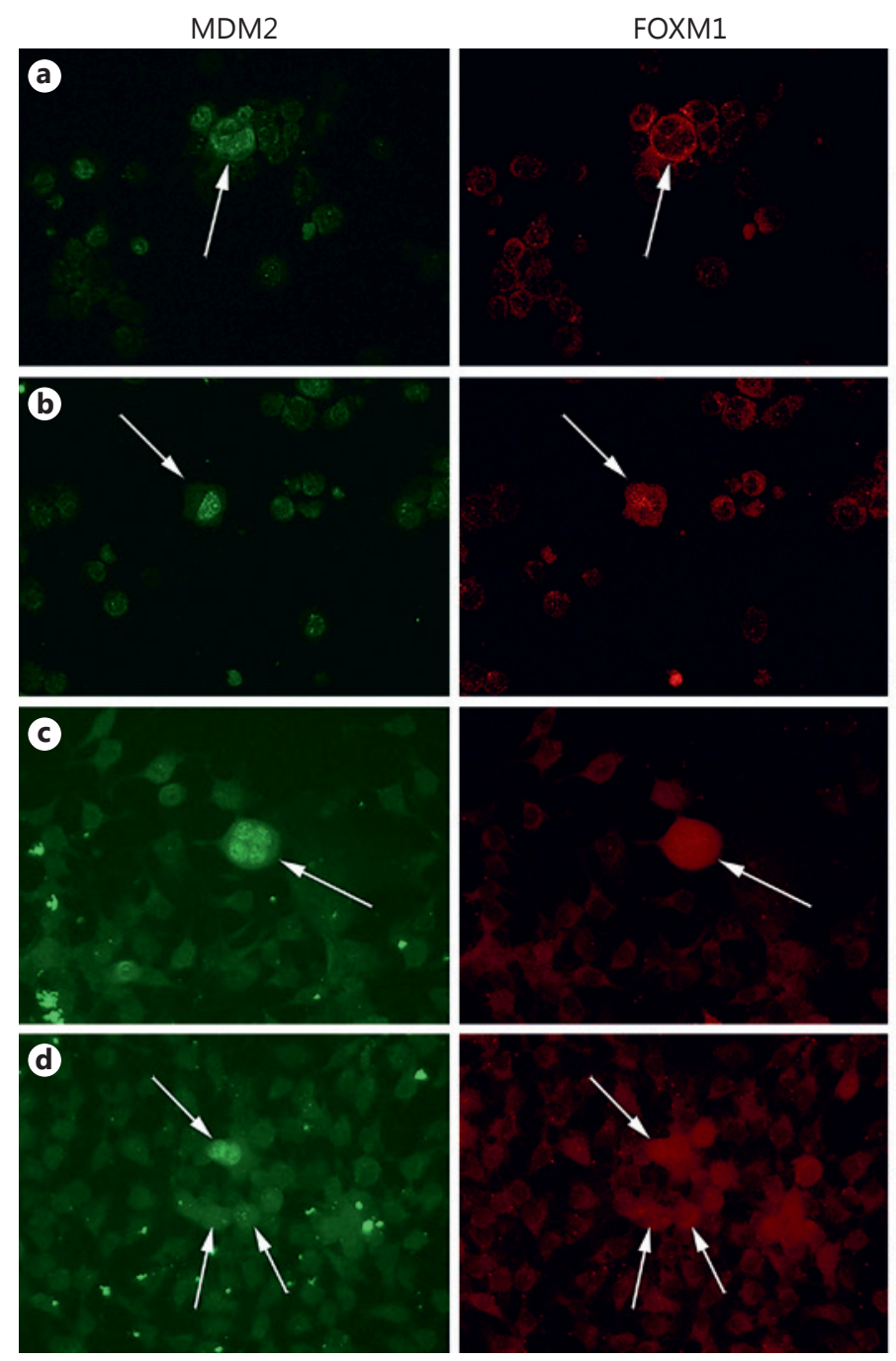

Fig. 10. Coexpression of MDM2 and FOXM1. Immunofluorescent costaining of MDM2 (green) and FOXM1 (red) in cytospin-placed $\mathrm{KRJ}-\mathrm{I}(\mathbf{a}, \mathbf{b})$ and BON-1 (c, d) cells revealed a coexpression in both cell lines. Especially very aberrant multinucleated cells showed a strong expression of both proteins $(\mathbf{a}, \mathbf{c})$. There is further evidence
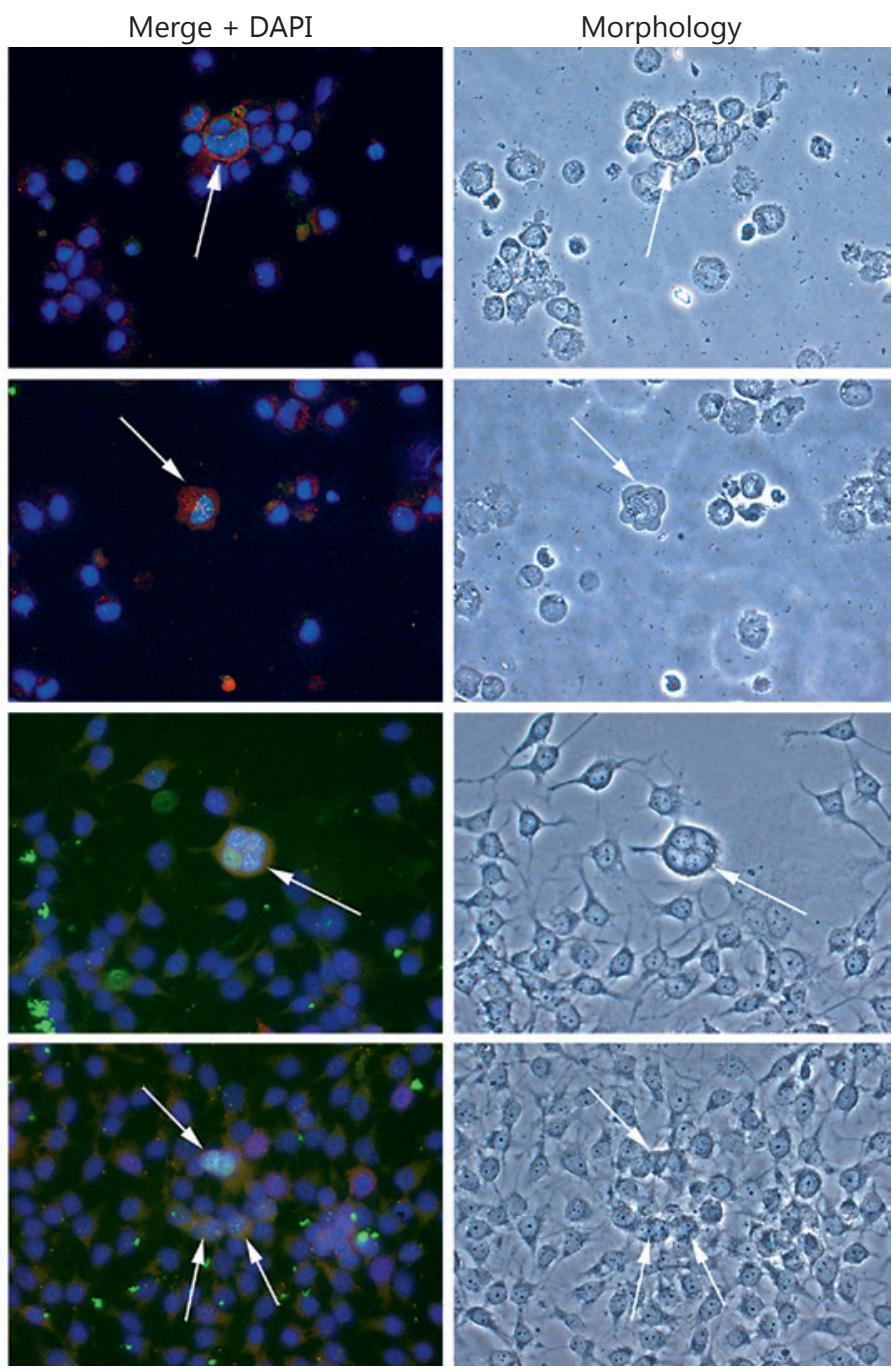

that FOXM1 was exclusively found in the cytoplasm in the wildtype KRJ-I cell line, whereas in the p53 mutated BON cells FOXM1 was detected in both the nucleus and the cytoplasm. Fluorescence microscopy. Magnification $\times 400$.
Fig. 11. Regulation of FOXM1 by MDM2. QGP-1 and KRJ-I cells were treated in independent experiments with 2 different siRNAs against MDM2 (or control siRNA) for $24 \mathrm{~h}$ and subsequently treated with nutlin-3a or DMSO for $72 \mathrm{~h}$. Representative data of 1 knockdown experiment are shown. a Treatment of KRJ-I cells with $5 \mu \mathrm{M}$ nutlin-3a for $72 \mathrm{~h}$ resulted in a $\mathrm{p} 53$-mediated increased expression of MDM2 and p21 and a decrease in FOXM1, FOXM1 activation and expression of the FOXM1 targets aurora A and SKP2. Here, the impact of MDM2 knockdown was negligible, as the effects were $\mathrm{p} 53$ driven and compensated by MDM2 re-expression. In contrast, p53-deficient QGP-1 cells did not induce a nut- lin-3a response, but $M D M 2$ knockdown resulted in a decrease in FOXM1 protein abundance, FOXM1 activation and target gene expression. Therefore, these effects must be independent from p53 activity. Blots were cropped to improve conciseness. Between lanes 1 and 2 of QGP-1, one empty lane was cut out. b Confirmation of the KRJ-I Western blot results by quantitative PCR: nutlin-3a for $72 \mathrm{~h}$ induced a strong expression of $\mathrm{p} 21$ and MDM2 mRNA, but a strong reduction of FOXM1 and its isoform FOXM1. c, d Densitometric quantification of all repeated Western blot experiments with different siRNAs. ${ }^{*} p<0.05$, related to siRNA control.

(For figure see next page.)
Briest et al. 
a

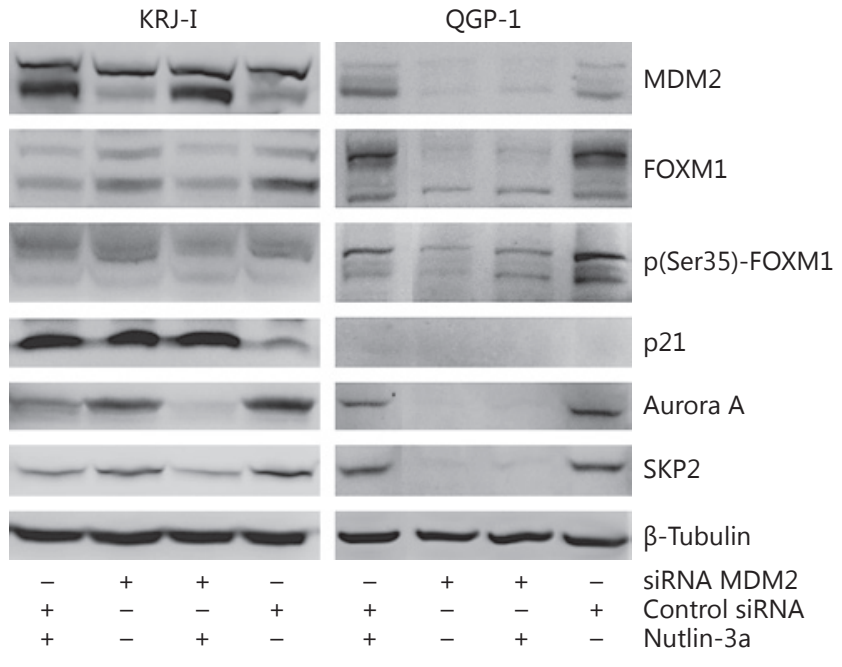

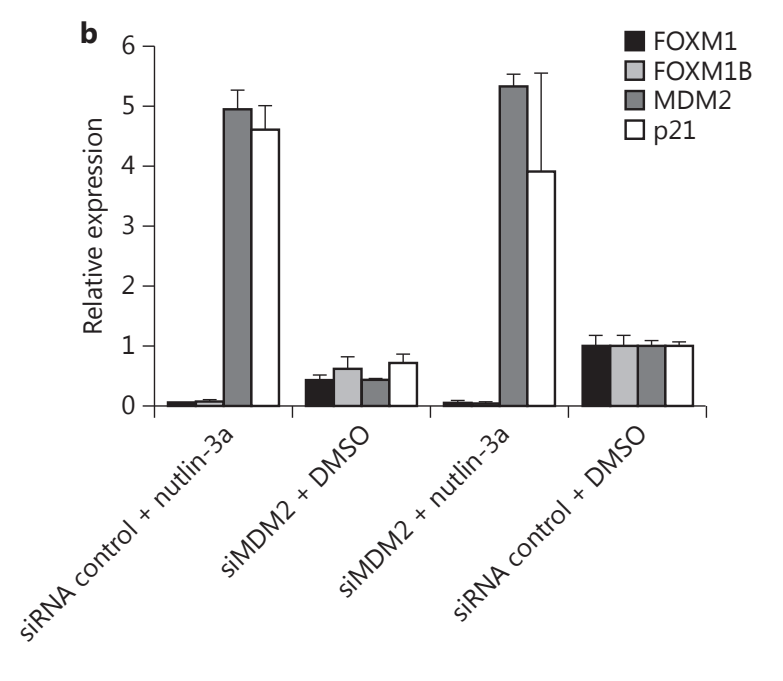
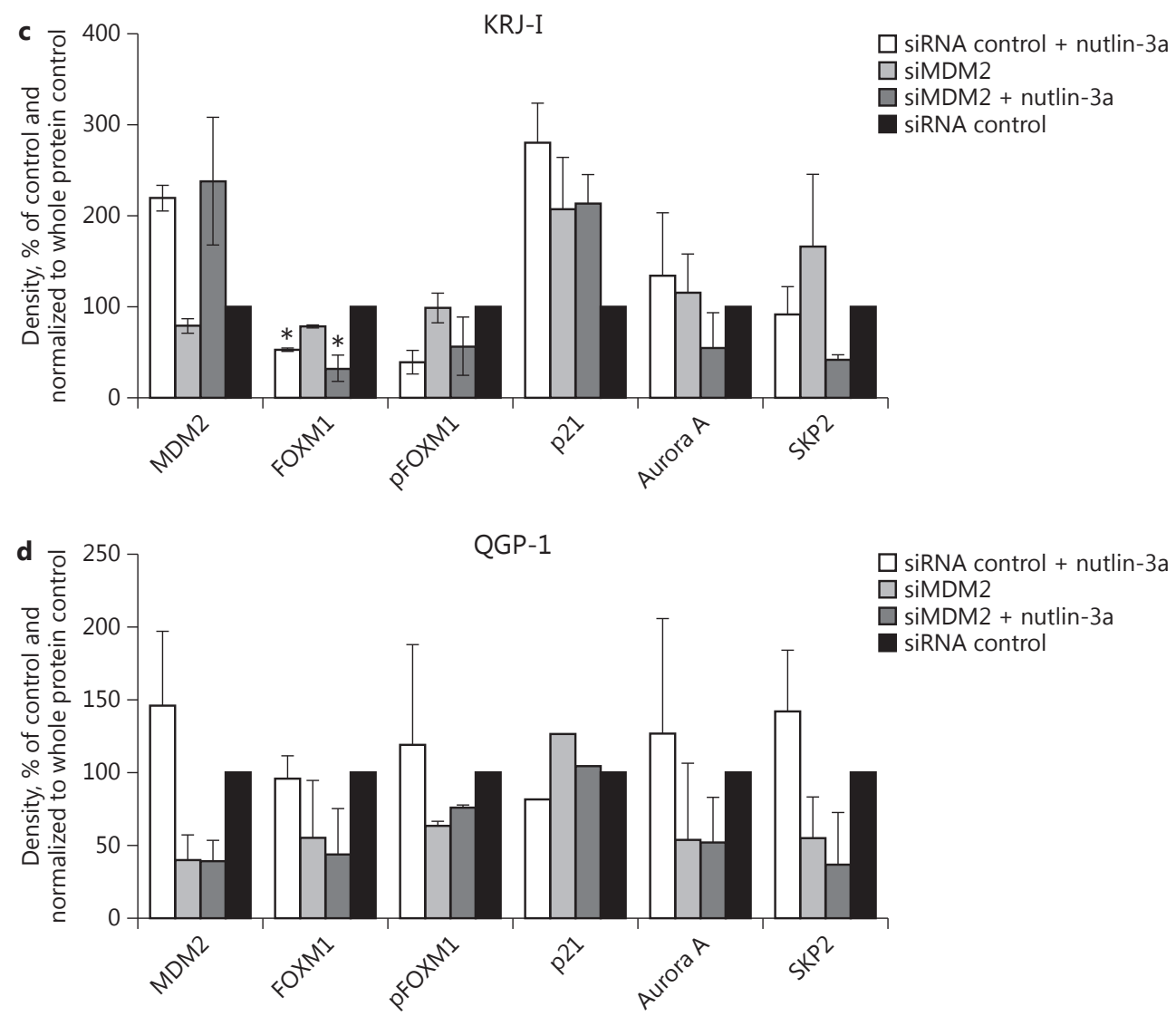


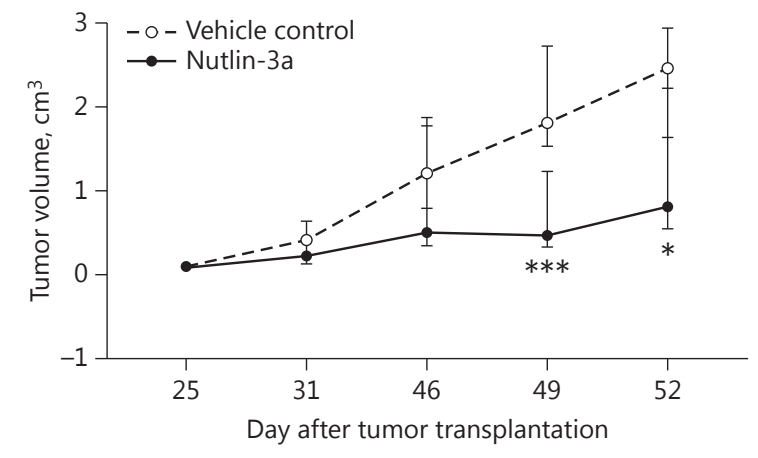

Fig. 12. Growth of xenograft tumors upon treatment with nutlin$3 \mathrm{a}$ in vivo. The study including $n=8 \mathrm{KRJ}$-I xenograft tumors per treatment group revealed antitumor activity of nutlin-3a in the KRJ-I tumor model. This is reflected by the optimal percent treated/control value (optT/C value) of $33.9 \%$ at study day 49 . The tumor growth is significantly reduced in the nutlin-3a group related to the vehicle control group (median with interquartile range is shown in the diagram; ANOVA: $p<0.0001)$. ${ }^{*} p<0.05,{ }^{* * *} p<$ 0.001 , related to vehicle control.

been shown to be inactivated after cisplatin-induced DNA damage, e.g. by ATM/ATR-mediated phosphorylation. Following ATM activation, the checkpoint kinase CHK2 further induces Ser20 phosphorylation of p53, which weakens the inhibitory interaction of p53 and MDM2 [38]. The intact $\mathrm{p} 53$ protein is stabilized and able to induce cell cycle arrest and apoptosis.

Therefore, the mechanisms why GEP-NEN do not respond to cisplatin are speculative and we assumed that the p53 target FOXM1 is involved in the regulation of resistance by induction of DNA repair. A combinatory effect has been described to be effective in several other cancer entities [26-28, 39, 40]. Furthermore, Reuther et al. [41] have recently demonstrated analogous effects concerning antiproliferative and chemosensitizing effects of another MDM2 antagonist, NVP-CGM097, on the p53 wild-type GOT-1 cell line, to which unfortunately only a limited number of research groups have access.

Chemotherapy is recommended as palliative therapy for metastatic foregut and pancreatic G2 and G3 NET [42-44]. Therefore, a combined treatment might be tailored for enhancing chemotherapy of progressive, higher proliferative p53 wild-type NET, where therapeutic options are a medical need (especially in midgut NET). Since functional p53 also represses the expression of MGMT (O6-methylguanine-DNA methyltransferase)
[45], which is a predictive marker for temozolomide treatment response at least in pancreatic NEN [46], the assessment of MDM2 inhibitors for enhancement of alkylating chemotherapy in progressive foregut and midgut NEN, should be further preclinically assessed. As p53 retrieval by nutlin-3a has been shown to further sensitize to radiotherapy [47], the potential of a reactivated p53 signaling should also be evaluated combined to other DNA-damaging regimens, peptide receptor radionuclide therapy and radiation.

Nutlin-3a treatment of KRJ-I cells resulted in a decreased proliferation in vitro and in vivo, induction of apoptosis and significant differential expression of a great number of known p53 and FOXM1 target genes. We could therefore verify the induction of a p53 response using the nCounter ${ }^{\circledR}$ gene expression analysis.

The gene expression pattern after the treatment suggests a functional shift characterized by decreased expression of DNA damage repair genes toward cell cycle arrest and apoptosis. Here, the proapoptotic effect seems to be an early event, which we were able to detect after $20 \mathrm{~h}$ of treatment by FACS analysis of depolarized mitochondrial membranes and after $72 \mathrm{~h}$ by detection of dead cells (sub-G1 cell population). Nevertheless, proapoptotic signaling was no longer clearly detectable in the Western blot analysis after $72 \mathrm{~h}$ and the gene expression analysis after $60 \mathrm{~h}$. As apoptosis is rather characterized by molecule cleavage and posttranslational modification than by de novo gene expression, we focused on the Western blot data. Here, we were able to show only a slight caspase 8 and BID cleavage as well as upregulated BAX, but no PARP or caspase 9 cleavage after long-term nutlin-3a treatment. This indicates that the majority of the cells was already in the late apoptotic state, where the signaling of the cells changes towards a secondary proinflammatory state [48] and where cell membranes are permeable for soluble proteins (which might therefore be lost for Western blot detection). Indeed, the PANTHER pathway anal$y$ sis of the differential expressed genes after $60 \mathrm{~h}$ of nutlin3a treatment resulted in a 6.1-fold overrepresentation of "cytokine-mediated signaling pathway" genes. Hence, an inflammatory secondary cell death expression pattern could be detected after long-term treatment. Nutlin-3a therefore induces an early apoptotic response in up to $50 \%$ of the cells after $24 \mathrm{~h}$ of treatment, whereas the remaining cells display the gene expression signature of cell cycle arrest. The conclusion that cell cycle arrest is the predominant response mechanism is supported by the fact that the xenograft tumors did not show a remission but a decelerated growth behavior. 
In detail, transcriptional p53 targets, which were strongly affected by nutlin-3a include replication-triggering genes, e.g. of the minichromosome maintenance complex (MCM proteins), which is part of the eukaryotic replicative helicase, regulated by FOXM1 [49-51] and have further been recently described to be relevant in GEP-NEN progression [52].

Interestingly, two further genes that have recently been described in GEP-NEN pathology were affected by nutlin-3a therapy: stathmin, which is a novel proliferation marker in pancreatic NEN [53] and upregulated by FOXM1 [54], was downregulated after nutlin treatment to a very high extent (approx. 9-fold). Further, the FOXM1 target and wnt antagonist AXIN2 [50], which has been found transcriptionally silenced in NEN cell lines [55], was found upregulated after nutlin-3a treatment (online suppl. Table 3).

Many genes that are critically involved in DNA repair and damage response and effectors of FOXM1, including CHEK1, BRCA1 and 2, BRIP1, RAD5 [56], of MSH and FANC proteins, SUV39H2 or PRKDC (DNA-PK catalytic subunit), were downregulated. This effect might result in higher sensitivity to cisplatin. Enhanced DNA repair is a feature of FOXM1-overexpressing cells and contributes to resistance to genotoxic agents [50]. Inhibition of DNA repair proteins sensitizes to alkylating or crosslinking chemotherapy and thus induces apoptosis or cell cycle arrest. As FOXM1 and mediators of several DNA repair pathways are downregulated under nutlin-3a treatment, we conclude that nutlin-3a might be able to target FOXM1-induced chemotherapy resistance and induce DNA damage stress as proven by enhanced histone H2AX phosphorylation.

We have previously described the p53 target FOXM1 as novel oncogenic player in gastrointestinal NEN [17]. FOXM1 is involved in mitosis regulation, metastasis, and genotoxic therapy resistance and is coexpressed with MDM2 in GEP-NEN cell lines. The fact that we found FOXM1 regulated by MDM2, dependent on and inde- pendent from p53, is interesting and remains to be explored. The fact further indicates that a more general, e.g. allosteric, MDM2 inhibitor might be more global and, thus, more beneficial.

Nutlins and further MDM2 antagonists have been described as potent inducers of cell cycle arrest and apoptosis in several preclinical cancer models $[39,57-60]$ and have entered clinical trials. We conclude that nutlin-mediated MDM2 antagonism can induce both cell cycle arrest and apoptosis in GEP-NEN by p53-dependent repression of cell cycle and DNA repair-related genes and FOXM1 repression on at least the transcriptional and posttranslational level. Additional in vivo verification of these results is necessary for further translational approaches, and further TP53 wild-type GEP-NEN cell lines remain to be generated for additional testing.

In summary, recovery of a wild-type p53 response in advanced intestinal NEN might be an effective new treatment possibility with the potential to sensitize to chemotherapies. Since effective treatment options in midgut NEN are limited, this approach should be further assessed for p53 wild-type tumors.

\section{Acknowledgments}

The authors would like to thank the colleagues of the Theranostics Research Network, especially Prof. Dieter Hörsch (Zentralklinik Bad Berka $\mathrm{GmbH}$ ) for providing specialist advice and support. The financial support of the Theranostics Research Network and the Sonnenfeld Stiftung Berlin is gratefully acknowledged.

\section{Disclosure Statement}

F.B. received a young researchers' grant by the Charité Universitätsmedizin Berlin. The group of Dr. Patricia Grabowski receives financial support from Ipsen Pharma, Novartis, and Pfizer. F.B. and P.G. received travel grants and lecture salaries from Ipsen Pharma. D.K. received travel support by Ipsen Pharma and Pfizer.

\section{References}

1 Modlin IM, Oberg K, Chung DC, Jensen RT, de Herder WW, Thakker RV, Caplin M, Delle Fave G, Kaltsas GA, Krenning EP, Moss SF, Nilsson O, Rindi G, Salazar R, Ruszniewski P, Sundin A: Gastroenteropancreatic neuroendocrine tumours. Lancet Oncol 2008;9:6172 .
2 Strosberg JR: Systemic treatment of gastroenteropancreatic neuroendocrine tumors (GEP-NETs): current approaches and future options. Endocr Pract 2014;20:167-175.

3 Briest F, Grabowski P: PI3K-AKT-mTORsignaling and beyond: the complex network in gastroenteropancreatic neuroendocrine neoplasms. Theranostics 2014;4:336-365.

4 Lane DP: Cancer. P53, guardian of the genome. Nature 1992;358:15-16. 
5 Grabowski P, Schrader J, Wagner J, Hörsch D, Arnold R, Arnold CN, Georgieva I, Stein H, Zeitz M, Daniel PT, Sturm I: Loss of nuclear p27 expression and its prognostic role in relation to cyclin E and p53 mutation in gastroenteropancreatic neuroendocrine tumors. Clin Cancer Res 2008;14:7378-7384.

6 Rindi G, Candusso ME, Solcia E: Molecular aspects of the endocrine tumours of the pancreas and the gastrointestinal tract. Ital J Gastroenterol Hepatol 1999;31(suppl 2):S135S138.

7 Lohmann DR, Funk A, Niedermeyer HP, Haupel S, Hofler H: Identification of p53 gene mutations in gastrointestinal and pancreatic carcinoids by nonradioisotopic SSCA. Virchows Arch B Cell Pathol Incl Mol Pathol 1993;64:293-296.

8 Weckstrom P, Hedrum A, Makridis C, Akerstrom G, Rastad J, Scheibenpflug L, Uhlen M, Juhlin C, Wilander E: Midgut carcinoids and solid carcinomas of the intestine: differences in endocrine markers and p53 mutations. Endocr Pathol 1996;7:273-279.

$9 \mathrm{Hu}$ W, Feng Z, Modica I, Klimstra DS, Song L, Allen PJ, Brennan MF, Levine AJ, Tang LH: Gene amplifications in well-differentiated pancreatic neuroendocrine tumors inactivate the p53 pathway. Genes Cancer 2010;1:360-368.

10 Ogawara Y, Kishishita S, Obata T, Isazawa Y, Suzuki T, Tanaka K, Masuyama N, Gotoh Y: Akt enhances mdm2-mediated ubiquitination and degradation of p53. J Biol Chem 2002;277:21843-21850.

11 Liu L, Broaddus RR, Yao JC, Xie S, White JA, Wu TT, Hamilton SR, Rashid A: Epigenetic alterations in neuroendocrine tumors: methylation of Ras-association domain family 1 , isoform A and p16 genes are associated with metastasis. Mod Pathol 2005;18:1632-1640.

12 Song MS, Song SJ, Kim SY, Oh HJ, Lim DS: The tumour suppressor RASSF1A promotes MDM2 self-ubiquitination by disrupting the MDM2-DAXX-HAUSP complex. EMBO J 2008;27:1863-1874.

13 Brazina J, Svadlenka J, Macurek L, Andera L, Hodny Z, Bartek J, Hanzlikova H: DNA damage-induced regulatory interplay between DAXX, p53, ATM kinase and Wip1 phosphatase. Cell Cycle 2015;14:375-387.

14 Briest F, Grabowski P: The p53 network as therapeutic target in gastroenteropancreatic neuroendocrine neoplasms. Cancer Treat Rev 2015;41:423-430.

15 Barsotti AM, Prives C: Pro-proliferative FOXM1 is a target of p53-mediated repression. Oncogene 2009;28:4295-4305.

16 Pandit B, Halasi M, Gartel AL: p53 negatively regulates expression of FoxM1. Cell Cycle 2009;8:3425-3427.

17 Briest F, Berg E, Grass I, Freitag H, Kaemmerer D, Lewens F, Christen F, Arsenic R, Altendorf-Hofmann A, Kunze A, Sänger J, Knösel T, Siegmund B, Hummel M, Grabowski P: FOXM1: a novel drug target in gastroenteropancreatic neuroendocrine tumors. Oncotarget 2015;6:8185-8199.
18 Zona S, Bella L, Burton MJ, Nestal de Moraes G, Lam EW: FOXM1: an emerging master regulator of DNA damage response and genotoxic agent resistance. Biochim Biophys Acta 2014; 1839:1316-1322.

19 Evers BM, Townsend CM Jr, Upp JR, Allen E, Hurlbut SC, Kim SW, Rajaraman S, Singh P, Reubi JC, Thompson JC: Establishment and characterization of a human carcinoid in nude mice and effect of various agents on tumor growth. Gastroenterology 1991;101:303311.

20 Kaku M, Nishiyama T, Yagawa K, Abe M: Establishment of a carcinoembryonic antigenproducing cell line from human pancreatic carcinoma. Gan 1980;71:596-601.

21 Pfragner R, Wirnsberger G, Niederle $B$, Behmel A, Rinner I, Mandl A, Wawrina F, Luo J, Adamiker D, Hoger H, Ingolic E, Schauenstein K: Establishment of a continuous cell line from a human carcinoid of the small intestine (KRJ-I). Int J Oncol 1996;8: 513-520.

22 Lundqvist M, Mark J, Funa K, Heldin NE, Morstyn G, Wedell B, Layton J, Öberg K: Characterisation of a cell line (LCC-18) from a cultured human neuroendocrine-differentiated colonic carcinoma. Eur J Cancer 1991;27: 1663-1668.

23 Chou TC: Theoretical basis, experimental design, and computerized simulation of synergism and antagonism in drug combination studies. Pharmacol Rev 2006;58:621-681.

24 http://www.combosyn.com/, 2015.

25 Calhoun S, Daggett V: Structural effects of the $1145 \mathrm{q}, \mathrm{v} 157 \mathrm{f}$, and $\mathrm{r} 282 \mathrm{w}$ cancer-associated mutations in the p53 DNA-binding core domain. Biochemistry 2011;50:5345-5353.

26 Barbieri E, Mehta P, Chen Z, Zhang L, Slack A, Berg S, Shohet JM: MDM2 inhibition sensitizes neuroblastoma to chemotherapy-induced apoptotic cell death. Mol Cancer Ther 2006;5:2358-2365.

27 Mir R, Tortosa A, Martinez-Soler F, Vidal A, Condom E, Perez-Perarnau A, Ruiz-Larroya T, Gil J, Gimenez-Bonafe P: MDM2 antagonists induce apoptosis and synergize with cisplatin overcoming chemoresistance in TP53 wild-type ovarian cancer cells. Int J Cancer 2013;132:1525-1536.

28 Roh JL, Kang SK, Minn I, Califano JA, Sidransky D, Koch WM: p53-reactivating small molecules induce apoptosis and enhance chemotherapeutic cytotoxicity in head and neck squamous cell carcinoma. Oral Oncol 2011; 47:8-15.

29 Kwok JM, Peck B, Monteiro LJ, Schwenen HD, Millour J, Coombes RC, Myatt SS, Lam EW: FOXM1 confers acquired cisplatin resistance in breast cancer cells. Mol Cancer Res 2010;8:24-34.

30 Liu Y, Chen X, Gu Y, Zhu L, Qian Y, Pei D, Zhang W, Shu Y: FOXM1 overexpression is associated with cisplatin resistance in non-small cell lung cancer and mediates sensitivity to cisplatin in A549 cells via the $\mathrm{JNK} /$ mitochondrial pathway. Neoplasma 2015;62:61-71.
31 Wang Y, Wen L, Zhao SH, Ai ZH, Guo JZ, Liu WC: FOXM1 expression is significantly associated with cisplatin-based chemotherapy resistance and poor prognosis in advanced nonsmall cell lung cancer patients. Lung Cancer 2013;79:173-179.

32 Fortina P, Surrey S: Digital mRNA profiling. Nat Biotechnol 2008;26:293-294.

33 Sbisà E, Catalano D, Grillo G, Licciulli F, Turi A, Liuni S, Pesole G, De Grassi A, Caratozzolo MF, D’Erchia AM, Navarro B, Tullo A, Saccone C, Gisel A: p53FamTaG: a database resource of human p53, p63 and p73 direct target genes combining in silico prediction and microarray data. BMC Bioinformatics 2007;8:S20

34 Yu H, Yue X, Zhao Y, Li X, Wu L, Zhang C, Liu Z, Lin K, Xu-Monette ZY, Young KH, Liu J, Shen Z, Feng Z, Hu W: LIF negatively regulates tumour-suppressor p53 through Stat3/ ID1/MDM2 in colorectal cancers. Nat Commun 2014;5:5218.

35 Laoukili J, Alvarez M, Meijer LA, Stahl M, Mohammed S, Kleij L, Heck AJ, Medema RH: Activation of FoxM1 during G2 requires cyclin A/Cdk-dependent relief of autorepression by the FoxM1 N-terminal domain. Mol Cell Biol 2008;28:3076-3087.

36 Meraldi P, Honda R, Nigg EA: Aurora-A overexpression reveals tetraploidization as a major route to centrosome amplification in p53-/- cells. EMBO J 2002;21:483-492.

37 Kelland LR: Preclinical perspectives on platinum resistance. Drugs 2000;59(suppl 4):1-8; discussion 37-38.

38 Yang J, Xu ZP, Huang Y, Hamrick HE, Duerksen-Hughes PJ, Yu YN: ATM and ATR: sensing DNA damage. World J Gastroenterol 2004;10:155-160.

39 Endo S, Yamato K, Hirai S, Moriwaki T, Fukuda K, Suzuki H, Abei M, Nakagawa I, Hyodo I: Potent in vitro and in vivo antitumor effects of MDM2 inhibitor nutlin-3 in gastric cancer cells. Cancer Sci 2011;102:605-613.

40 Koster R, Timmer-Bosscha H, Bischoff R, Gietema JA, de Jong S: Disruption of the MDM2-p53 interaction strongly potentiates p53-dependent apoptosis in cisplatin-resistant human testicular carcinoma cells via the Fas/FasL pathway. Cell Death Dis 2011;2: e148.

41 Reuther C, Heinzle V, Nölting S, Herterich S, Hahner S, Halilovic E, Jeay S, Wuerthner JU, Aristizabal Prada ET, Spottl G, Maurer J, Auernhammer CJ: The HDM2 (MDM2) inhibitor NVP-CGM097 inhibits tumor cell proliferation and shows additive effects with 5-fluorouracil on the p53-p21 - Rb - E2F1 cascade in the p53wildtype neuroendocrine tumor cell line GOT1. Neuroendocrinology 2016, Epub ahead of print.

42 Strosberg JR, Coppola D, Klimstra DS, Phan AT, Kulke MH, Wiseman GA, Kvols LK: The NANETS consensus guidelines for the diagnosis and management of poorly differentiated (high-grade) extrapulmonary neuroendocrine carcinomas. Pancreas 2010;39:799-800. 
43 Öberg K, Knigge U, Kwekkeboom D, Perren A: Neuroendocrine gastro-entero-pancreatic tumors: ESMO clinical practice guidelines for diagnosis, treatment and follow-up. Ann Oncol 2012;23(suppl 7):vii124- vii130.

44 Pavel M, Baudin E, Couvelard A, Krenning E, Öberg K, Steinmuller T, Anlauf M, Wiedenmann B, Salazar R: ENETS consensus guidelines for the management of patients with liver and other distant metastases from neuroendocrine neoplasms of foregut, midgut, hindgut, and unknown primary. Neuroendocrinology 2012;95:157-176.

45 Srivenugopal KS, Shou J, Mullapudi SR, Lang FF Jr, Rao JS, Ali-Osman F: Enforced expression of wild-type p53 curtails the transcription of the $\mathrm{O}(6)$-methylguanine-DNA methyltransferase gene in human tumor cells and enhances their sensitivity to alkylating agents. Clin Cancer Res 2001;7:1398-1409.

46 Schmitt AM, Pavel M, Rudolph T, Dawson $\mathrm{H}$, Blank A, Komminoth P, Vassella E, Perren A: Prognostic and predictive roles of MGMT protein expression and promoter methylation in sporadic pancreatic neuroendocrine neoplasms. Neuroendocrinology 2014;100:3544.

47 Impicciatore G, Sancilio S, Miscia S, Di Pietro $R$ : Nutlins and ionizing radiation in cancer therapy. Curr Pharm Des 2010;16:14271442.

48 Galluzzi L, Bravo-San Pedro JM, Vitale I, Aaronson SA, Abrams JM, Adam D, Alnemri ES, Altucci L, Andrews D, Annicchiarico-Petruzzelli M, Baehrecke EH, Bazan NG, Bertrand MJ, Bianchi K, Blagosklonny MV, Blomgren $\mathrm{K}$, Borner C, Bredesen DE, Brenner C, Cam- panella M, Candi E, Cecconi F, Chan FK, Chandel NS, Cheng EH, Chipuk JE, Cidlowski JA, Ciechanover A, Dawson TM, et al: Essential versus accessory aspects of cell death: recommendations of the NCCD 2015. Cell Death Diff 2015;22:58-73.

49 Vijayraghavan S, Schwacha A: The eukaryotic MCM2-7 replicative helicase. Subcell Biochem 2012;62:113-134.

50 Wierstra I: FOXM1 (Forkhead box M1) in tumorigenesis: overexpression in human cancer, implication in tumorigenesis, oncogenic functions, tumor-suppressive properties, and target of anticancer therapy. Adv Cancer Res 2013;119:191-419.

51 Lefebvre C, Rajbhandari P, Alvarez MJ, Bandaru P, Lim WK, Sato M, Wang K, Sumazin P, Kustagi M, Bisikirska BC, Basso K, Beltrao P, Krogan N, Gautier J, Dalla-Favera R, Califano A: A human B-cell interactome identifies MYB and FOXM1 as master regulators of proliferation in germinal centers. Mol Syst Biol 2010;6:377.

52 Schimmack S, Lawrence B, Kenney B, Schmitz-Winnenthal H, Modlin IM, Kidd M: Minichromosome maintenance expression defines slow-growing gastroenteropancreatic neuroendocrine neoplasms. Transl Oncol 2016;9:411-418.

53 Schimmack S, Taylor A, Lawrence B, SchmitzWinnenthal H, Fischer L, Buchler MW, Modlin IM, Kidd M, Tang LH: Stathmin in pancreatic neuroendocrine neoplasms: a marker of proliferation and PI3K signaling. Tumour Biol 2015;36:399-408.

54 Park HJ, Gusarova G, Wang Z, Carr JR, Li J, Kim KH, Qiu J, Park YD, Williamson PR, Hay
N, Tyner AL, Lau LF, Costa RH, Raychaudhuri P: Deregulation of FOXM1B leads to tumour metastasis. EMBO Mol Med 2011;3: 21-34.

55 Kim JT, Li J, Jang ER, Gulhati P, Rychahou PG, Napier DL, Wang C, Weiss HL, Lee EY, Anthony L, Townsend CM Jr, Liu C, Evers BM: Deregulation of wnt/beta-catenin signaling through genetic or epigenetic alterations in human neuroendocrine tumors. Carcinogenesis 2013;34:953-961.

56 Lam EW, Brosens JJ, Gomes AR, Koo CY: Forkhead box proteins: tuning forks for transcriptional harmony. Nat Rev Cancer 2013; 13:482-495.

57 Henze J, Mühlenberg T, Simon S, Grabellus F, Rubin B, Taeger G, Schuler M, Treckmann J, Debiec-Rychter M, Taguchi T, Fletcher JA, Bauer S: p53 modulation as a therapeutic strategy in gastrointestinal stromal tumors. PLoS One 2012;7:e37776.

58 Park EJ, Choi KS, Yoo YH, Kwon TK: Nutlin-3, a small-molecule MDM2 inhibitor, sensitizes Caki cells to TRAIL-induced apoptosis through p53-mediated Puma upregulation and ROS-mediated DR5 upregulation. Anticancer Drugs 2013;24:260-269.

59 Polanski R, Noon AP, Blaydes J, Phillips A, Rubbi CP, Parsons K, Vlatkovic N, Boyd MT: Senescence induction in renal carcinoma cells by nutlin-3: a potential therapeutic strategy based on MDM2 antagonism. Cancer Lett 2014;353:211-219.

60 Qin L, Yang F, Zhou C, Chen Y, Zhang H, Su $\mathrm{Z}$ : Efficient reactivation of $\mathrm{p} 53$ in cancer cells by a dual MDMX/MDM2 inhibitor. J Am Chem Soc 2014;136:18023-18033. 